\title{
Job Market Signaling and Employer Learning*
}

\author{
Carlos Alós-Ferrer ${ }^{\dagger}$ and Julien Prat ${ }^{\ddagger}$ \\ Revised version: February 2010
}

\begin{abstract}
We consider a signaling model where the receiver is able to update his belief about the sender's type after the signaling stage. We introduce Bayesian learning in a variety of environments ranging from simple two-period to continuous time models with stochastic production. Signaling equilibria present two major departures from those obtained in models without learning. First, new mixedstrategy equilibria involving multiple pooling are possible. Second, pooling equilibria can survive the Intuitive Criterion when learning is fast enough.
\end{abstract}

\section{Introduction}

Signaling models are concerned with situations where an agent is able to send messages about information that he could not otherwise credibly reveal. Private information is valuable to the extent that it helps to predict the outcome of the transaction between the sender and the receiver. When the relationship involves repeated interactions, observing each outcome allows the receiver to update his belief. He is therefore able to gather knowledge about the sender after the signaling stage, a possibility that is usually excluded in signaling games. We argue that this omission is not inconsequential by embedding Bayesian learning in a standard signaling model and establishing that: (i) qualitatively new equilibria emerge; and (ii) forward induction (as captured by the Intuitive Criterion) loses part of its predictive power.

These features arise because expectations about future payoffs do not solely depend on the equilibrium belief, as is commonly assumed in the literature, but also on the sender's type. Even when agents

\footnotetext{
${ }^{*}$ We are grateful to three anonymous refereees, Egbert Dierker, Gerhard Orosel, Larry Samuelson, and Gerhard Sorger, as well as seminar participants in Brussels, Hamburg, Konstanz, and Vienna, and conference participants at the ESEM and SED 2007 meetings for helpful comments.

${ }^{\dagger}$ Department of Economics, University of Konstanz (Germany). Email: carlos.alos-ferrer@uni-konstanz.de

${ }^{\ddagger}$ Institute of Economic Analysis (IAE-CSIC), Barcelona (Spain). Email: julien.prat@iae.csic.es
} 
are indistinguishable right after the signaling stage, their expectations vary with their productivity because they know that it will be identified over time. The standard premise that senders are equally rewarded in pooling equilibria is therefore violated.

For the sake of concreteness, this general mechanism and its ramifications are illustrated in a jobmarket signaling model along the lines of Spence's (1973) seminal work. We consider an economy where workers are better informed about their ability than prospective employers. In order to signal their capacity, talented individuals have an incentive to invest in education. Alternatively, they may decide to save on educational costs and trust that their actual productivity will be revealed by performances on-the-job. Spence's model does not take into account this countervailing incentive because it assumes that all information is collected prior to labor market entry. Most of the ensuing theoretical literature follows Spence's approach and has ignored employer learning. ${ }^{1}$ Econometricians, on the other hand, have devised ingenious tests for unobservable characteristics in order to estimate the efficiency of signal extraction in labor markets. Empirical evidence documented in Lange (2007) shows that employers are not only able to elicit information about workers' abilities but that the speed at which they do so is quite fast, with nearly $95 \%$ of the statistically significant information being collected after solely 3 years. $^{2}$

This finding suggests that it is important to assess whether the outcomes of the signaling game are indeed unaffected by the learning process. We show in this paper that it is not the case. Firstly, qualitatively new types of equilibria may arise where more than one common level of education is acquired by both types of workers with positive probability (multiple pooling). Secondly, the standard refinement argument embodied in the Intuitive Criterion do not bite when employer learning is sufficiently fast.

The second observation is particularly relevant from a game-theoretic point of view. The mul-

\footnotetext{
${ }^{1}$ The paper by Gibbons and Katz (1991) is a notable exception. They do not focus on the game theoretical analysis but on the implications of asymmetric information for layoff decisions. Although not directly concerned with learning, the paper by Feltovich et al. (2002) also allows correlated information to be revealed after the signaling stage. They consider a set-up with three types and show that high types may pool with low types at the lowest level of education. More recently, Daley and Green (2007) have analyzed a signaling model with grades whose implications are discussed in Section 5 .

${ }^{2}$ Lange (2007) is among the latest contributions to a strand of literature measuring the speed of employer learning. The identifying assumption is that econometricians have access to a correlate of workers' abilities that is not available to employers (generally Armed Forces Qualification Test scores of workers). Farber and Gibbons (1996) and Altonji and Pierret (2001) document that the impact of this correlate on wages increases with labor market experience. Even though this result indicates that employers learn over time, it does not distinguish between symmetric and asymmetric learning and so does not test job market signaling. Nevertheless, as explained by Lange (2007), the estimated speed of employer learning can be used to place an upper bound on the contribution of signaling to the gains of schooling.
} 
tiplicity of equilibria in Spence's model has been a motivation for the vast literature on refinement concepts. For signaling games with only two types, the Intuitive Criterion of Cho and Kreps (1987) is the most commonly used refinement because it excludes all but one separating equilibrium. We prove that this does not always hold true when employers are able to update their beliefs. The key difference between the two environments is that learning yields higher asset values for talented individuals even when pooling is the equilibrium outcome. The gap increases with the speed of learning as low and high types become less and more optimistic, respectively. The stronger the correlation between a worker's ability and his observable performance, the more attractive it is for high types to reveal their ability on-the-job instead of paying the educational costs. ${ }^{3}$ This is why high types find it optimal to pool with low types when learning is fast.

We formalize this mechanism in a model which embeds Spence's signaling game into a dynamic framework with Bayesian learning on the side of firms. Workers of different abilities can acquire education before entering the labor market. We assume for simplicity that their abilities are either high or low. ${ }^{4}$ We extend the basic signaling model by allowing employers to update their beliefs, hence types are gradually revealed over time. Our set-up therefore bears similarities to Jovanovic's (1979) matching model with the crucial difference that uncertainty is not match-specific but worker-specific. In this respect, it bridges the gap between the theoretic literature on signaling games and the labor market literature.

The paper is organized as follows. Section 2 lays out the model's set-up. To underline the generality of the results, we initially adopt a reduced form approach. We define workers' value functions and specify, using intuitive arguments, which key properties they should fulfill in order to capture the learning process. Section 3 then illustrates how these properties can be derived from first principles, describing the signal extraction problem in examples with both discrete and continuous time settings. In Section 4, we analyze the equilibria (in pure and mixed strategies) of our model using general value functions. In Section 5, we discuss the conditions under which the Intuitive Criterion can refine the set of equilibria and explain why they are not met when employer learning is efficient enough. Section 6 concludes. Proofs are relegated to the Appendix.

\footnotetext{
${ }^{3}$ On the other hand, the incentive for low types to send misleading signals, and thus to acquire education, increases with the speed of employer learning. A recent working paper by Haberlmaz (2006) proposes a partial equilibrium model which underlies the ambiguity of the relationship between the value of job market signaling and the speed of employer learning.

${ }^{4}$ Allowing for more than two types leads to technical difficulties in the application of the Intuitive Criterion (see e.g. Cho and Kreps (1987)).
} 


\section{Signaling and Employer Learning}

Workers differ in their innate abilities. They can be of different types which determine their productivity. Nature initially selects types according to pre-specified probabilities. The main ingredient of the model is information asymmetry: Workers know their abilities whereas employers must infer them.

The game is as follows. In a first step, the worker chooses an education level. In a second step, the industry offers a starting wage based on beliefs derived from the education signal. In Spence's static framework, the game ends as the worker enters the labor market. In our set-up, in contrast, a third step is added, where the relationship develops, with the industry being able to extract information from noisy realizations of the worker's productivity. Rather than proposing a particular dynamic model, we adopt a reduced-form approach to employer learning and delay its micro-foundation to Section 3.

I. Education decision: We restrict our attention to cases where workers (senders) are of only two types, $i=h$ (high) or $i=l$ (low). Nature assigns a productivity $p \in\left\{p_{l}, p_{h}\right\}$ to the worker with $p_{h}>p_{l}$. High-types account for a share $\mu_{0}<1$ of the population. For simplicity, we assume that workers are infinitely lived and that they discount the future at the common rate $r$. Before entering the labor market, workers choose their education level $e \in[0,+\infty)$. To isolate the effect of signaling, we do not allow education to increase productivity. Its only use is to signal abilities which are initially unobserved by the industry (receiver).

Let the function $c: \mathbb{R}^{++} \times \mathbb{R}^{+} \rightarrow \mathbb{R}^{+}$specify the cost of acquiring education. That is, $c(p, e)$ is the cost that a worker with innate productivity $p$ has to pay in order to acquire education level $e .^{5}$ The cost function is twice differentiable with $c_{e}(p, e)>0$ and $c_{e e}(p, e) \geq 0$, hence strictly increasing and convex in the level of education. As commonly assumed in the literature, we also let $c_{p}(p, e)<0$ and $c_{p e}(p, e)<0$, so that total and marginal costs of education are strictly decreasing in ability. The last requirement ensures that low types have steeper indifference curves than high types because it implies the submodularity condition

$$
c\left(p_{h}, e^{\prime \prime}\right)-c\left(p_{h}, e^{\prime}\right)<c\left(p_{l}, e^{\prime \prime}\right)-c\left(p_{l}, e^{\prime}\right) \quad \text { whenever } e^{\prime \prime}>e^{\prime},
$$

which is commonly referred to as the Single Crossing Property.

II. Wage setting: As in Spence's model, a worker is paid his expected productivity. This wage setting rule is justified as a proxy for a competitive labor market or a finite number of firms engaged

\footnotetext{
${ }^{5}$ Even though workers will only have productivity levels in $\left\{p_{l}, p_{h}\right\}$, it is convenient to define the cost function for all potential productivities.
} 
in Bertrand competition for the services of the worker. Under the first possibility, one can make sense of the condition by assuming a single player in place of the industry, with payoffs given by a quadratic loss function $-(w-p)^{2}$. In any Perfect Bayesian Equilibrium, optimal behavior of this player will lead to a wage offer equal to the expected productivity. ${ }^{6}$ Under the second possibility, where Bertrand competition among multiple firms is explicitly introduced, the equilibrium concept must be refined to ensure that all firms share the same beliefs about the worker. ${ }^{7}$ Let $\mu(e)$, which we abbreviate by $\mu$ when no confusion may arise, denote the probability that the industry attaches to the worker being of the high type given education signal $e$. The initial wage is given by $w(\mu)=(1-\mu) p_{l}+\mu p_{h}$. We can therefore use the equilibrium belief of the industry to denote its response to a particular signal.

III. Expected income: The key departure from Spence's model is the third step where we specify the sender's expected income. We adopt in this section a reduced-form approach: Given the actual productivity $p$ and the employer's prior $\mu=\mu(e)$, expected earnings are given by a value function ${ }^{8}$ $v(p, \mu)$ which captures the impact that employer learning has on future income. ${ }^{9}$

For the purposes of enabling comparative statics, we explicitly introduce two additional parameters. The first one is the common discount rate $r$ of workers whose interpretation depends on the particular model behind the value function. We adopt the convention that the forward-discounted value is given by $w / r$ when the agent receives a constant wage $w$, as in a continuous-time model with $r>0$. The second parameter measures the speed of learning of the industry $s>0 .{ }^{10}$ Thus we will write $v(p, \mu \mid r, s)$ whenever we wish to discuss the effect of those two parameters.

Let us now specify the properties that should be imposed on value functions and the rationales

\footnotetext{
${ }^{6}$ Even though the resulting game is properly specified, there is no economic interpretation for the payoff function $-(w-p)^{2}$ but rather for the result of the optimization problem.

${ }^{7}$ This is the approach adopted by Mas-Colell et al (1995) in Section 13.C of their textbook. Notice that common beliefs follow from the concept of sequential equilibrium but not of Perfect Bayesian Equilibrium. However, sequential equilibrium is only defined for finite games.

${ }^{8}$ From a purely game-theoretic point of view, the value function needs to be interpreted as an equilibrium device, in the sense that, given a specific micro-foundation (i.e. a specification of the part of the game which the function summarizes), the computation of the outcome delivered by the function must rely on the explicit use of a uniquely defined equilibrium concept. As we will show in Section 3, alternative value functions can be obtained from different models.

${ }^{9}$ The value function depends on the industry's prior rather than the initial wage because, as commented before, we use the belief of the industry to denote its response to the education signal. This relies on the equilibrium requirement that beliefs uniquely determine wages. Strictly speaking, out-of-equilibrium behavior for the industry might involve wages which are not consistent with beliefs. This possibility, however, is inconsequential for the analysis because of the game structure. Alternatively, one can simply consider that Spence's modeling device, which yields a wage of $w(\mu)$ is replaced by $v(p, \mu)$ in our model.

${ }^{10} \mathrm{~A}$ micro foundation for the speed of learning is derived in the continuous time model of Section 3.
} 
behind them. As employment histories unfold, employers observe the cumulative outputs produced by workers and use this information to revise their priors. Consider how the updating process affects the expectations of low types. For every $\mu \in(0,1)$, they are offered an initial wage $w(\mu)$ that is above their actual productivity $p_{l}$. On average, realized outputs will induce the industry to lower its belief and thus wages. Their expected income $v\left(p_{l}, \mu\right)$ is therefore smaller than the forward discounted value of the starting wage $w / r$, but larger than the value $p_{l} / r$ that they would have obtained if the industry had known their type with certainty. A symmetric argument holds for high types because their initial wage is lower than their actual productivity. On the other hand, when industry's beliefs collapse to certainty, ${ }^{11}$ that is $\mu \in\{0,1\}$, further information will be ignored and the initial wage will never be altered. Since we want to encompass Spence's model as a particular case, we first introduce a weak implication of this argument:

P0. For all $\mu \in(0,1)$,

$$
\frac{p_{h}}{r}>v\left(p_{h}, \mu\right) \geq \frac{w(\mu)}{r} \geq v\left(p_{l}, \mu\right)>\frac{p_{l}}{r} .
$$

Further, when $\mu \in\{0,1\}, v\left(p_{h}, \mu\right)=v\left(p_{l}, \mu\right)=w(\mu) / r$.

Because of learning, we will actually expect a stronger variant of this property to hold:

P1. For all $\mu \in(0,1)$,

$$
\frac{p_{h}}{r}>v\left(p_{h}, \mu\right)>\frac{w(\mu)}{r}>v\left(p_{l}, \mu\right)>\frac{p_{l}}{r} .
$$

Further, when $\mu \in\{0,1\}, v\left(p_{h}, \mu\right)=v\left(p_{l}, \mu\right)=w(\mu) / r$.

Property P1 ensures that firms are able to update their beliefs towards the realization of the randomly assigned productivity. We will show in Section 3 that this is a general consequence of Bayesian learning.

Compare now two industries with different speeds of learning. Workers whose productivity is overestimated prefer being employed in the industry where signal extraction is slow. Conversely, workers whose productivity is underestimated would rather choose the industry where actual abilities are quickly recognized. The following property captures this intuition.

P2. For all $\mu \in(0,1)$, we have that $\partial v\left(p_{h}, \mu \mid r, s\right) / \partial s>0$ and $\partial v\left(p_{l}, \mu \mid r, s\right) / \partial s<0$, hence $v\left(p_{h}, \mu \mid r, s\right)$ is strictly increasing in $s$ whereas $v\left(p_{l}, \mu \mid r, s\right)$ is strictly decreasing in $s$.

\footnotetext{
${ }^{11}$ Observe that, since we are describing the game and not an equilibrium outcome, we do not assume that beliefs are correct and thus define the value function of the low (high) type when $\mu=1(\mu=0)$.
} 
We will show in Section 3 how to derive the speed of learning from primitive parameters describing the production process and prove that its impact on $v(\cdot)$ is as postulated in P2.

Finally, consider limit cases where learning is very slow or very fast. As the speed of learning goes to zero, employers have no possibility to update their initial beliefs: Wages are never revised and the value functions converge to their original specification in Spence's model. Conversely, when the speed of learning goes to infinity, types are immediately recognized and signaling becomes redundant.

P3. For all $\mu \in(0,1), \lim _{s \rightarrow 0} v(p, \mu \mid r, s)=w(\mu) / r$ and $\lim _{s \rightarrow \infty} v(p, \mu \mid r, s)=p / r$.

Definition 1. A value function is a mapping assigning a lifetime income $v(p, \mu)$ to each belief $\mu \in[0,1]$ and productivity $p \in\left\{p_{l}, p_{h}\right\}$. It is assumed to be strictly increasing and twice differentiable in $\mu$, and to fulfill property $P 0$.

A value function is said to exhibit weak learning if it fulfills the more demanding property P1. It exhibits strong learning if it fulfills properties P1, P2, and P3.

V. Equilibrium concept: The equilibrium concept is just Perfect Bayesian Equilibrium for the game as specified before. ${ }^{12}$ Combining the value and cost functions yields the payoff function for workers: $u(e, \mu \mid p) \equiv v(p, \mu)-c(p, e)$. As discussed in sub-section II, industry's payoffs are inconsequential as long as the specification leads to wage offers equal to the expected productivity given the beliefs.

We will consider equilibria in pure and mixed strategies ("hybrid equilibria"). The very formalization of both Spence's model and ours is such that the industry never has an incentive to randomize. Hence, an equilibrium in mixed strategies can be defined as follows.

A signaling equilibrium is made out of a pair of probability distributions $(q(\cdot \mid p))_{p \in\left\{p_{l}, p_{h}\right\}}$ on $\mathbb{R}^{+}$ describing the education levels chosen by both types, and a belief system $(\mu(e))_{e \in \mathbb{R}^{+}}$describing the priors of the industry given any possible signal, that satisfies the following properties:

- Sequential rationality for the workers: For $p \in\left\{p_{l}, p_{h}\right\}$, if $e^{*}$ belongs to the support of $q(\cdot \mid p)$, then $e^{*} \in \arg \max _{e \in \mathbb{R}^{+}} u(e, \mu(e) \mid p)$.

\footnotetext{
${ }^{12}$ There is some confusion in the older game-theoretic literature with respect to the Perfect Bayesian Equilibrium (PBE) concept. For games with the structure of signaling games, PBEs coincide with "Weak PBEs" which are defined as pairs of beliefs and strategy profiles such that actions at any information set maximize payoffs given the beliefs (sequential rationality) and beliefs are consistent with strategies through Bayes' rule along the equilibrium path (weak consistency). This is the equilibrium concept that the Intuitive Criterion aims to refine. Unfortunately, some of the earlier literature called PBEs "sequential" in reference to the sequential rationality requirement. The concept of sequential equilibrium (Kreps and Wilson 1982), however, is defined for finite games only and hence does not apply in our framework.
} 
- (Weak) consistency of beliefs: The industry's initial beliefs $\mu(e)$ are consistent with Bayes' rule for any educational attainment $e$ in the support of either $q\left(\cdot \mid p_{h}\right)$ or $q\left(\cdot \mid p_{l}\right)$.

- Sequential rationality for the industry: Given any education level $e$, the industry offers an initial wage equal to $w(\mu(e))$.

In the pure strategy case, the support of the distributions $q(\cdot \mid p)$ is a singleton and hence a signaling equilibrium in pure strategies can be described by a belief system as before, and a strategy profile $\left(e_{l}, e_{h}\right)$ describing the deterministic education levels chosen by both types. ${ }^{13}$

\section{Bayesian Learning}

Spence's model is encompassed by our model taking the value function $v(p, \mu)=w(\mu) / r$, with the interpretation that the starting wage is final and no further adjustments (of wages or beliefs) are possible. This function fulfills the basic conditions in Definition 1. Alternatively, it can be interpreted as a limit case of a value function with strong learning when $s$ goes to 0 .

This section shows that the stronger properties listed in Definition 1 not only conform to intuition but also follow from Bayesian learning. We propose three examples in order of increasing complexity.

\subsection{Reports model}

The first model is included mostly for illustrative purposes as it allows for considerably simpler computations. We assume that updating occurs only once over the labor market career of a given worker. Thus production can be divided in just two periods. The employer has access to a detection technology which, at the end of the first period, delivers a signal about the productivity of the worker. We call this signal a "report" in order to avoid confusion with the education decision. ${ }^{14}$

There are two possible reports, $\mathrm{G}(\mathrm{ood})$ and $\mathrm{B}(\mathrm{ad})$. If the productivity of the worker is high, a good report is delivered with probability $d(s)>1 / 2$ for $s \in(0,+\infty)$, and a bad report is delivered

\footnotetext{
${ }^{13}$ For the mixed-strategy case, we require workers to randomize among optimal education levels only. This eliminates from the onset technical difficulties associated with the inclusion of suboptimal strategies with zero probability in the support of the equilibrium strategies. The typical examples we have in mind at this point involve randomization over finitely many education levels. For such equilibria, belief consistency amounts to the assertion that, if there exists a $p \in\left\{p_{l}, p_{h}\right\}$ such that $q(e \mid p)>0$, then $\mu(e)=\mu_{0} q\left(e \mid p_{h}\right) /\left[\mu_{0} q\left(e \mid p_{h}\right)+\left(1-\mu_{0}\right) q\left(e \mid p_{l}\right)\right]$. A priori, however, an equilibrium strategy might prescribe a randomization over an infinite set.

${ }^{14}$ That is, actual production by the worker does not influence learning. A possible justification is that output cannot be traced back to each individual. However, we insist that this is merely a "toy model". The reader is referred to the following two sub-sections for more realistic micro-foundations.
} 
with the complementary probability. If the worker's productivity is low, the likelihoods of a good or bad report are $1-d(s)<\frac{1}{2}$ and $d(s)$, respectively. The function $d(s)$ is strictly increasing in $s$ with the following lower and upper bounds: $\lim _{s \rightarrow 0} d(s)=1 / 2$ and $\lim _{s \rightarrow \infty} d(s)=1$.

Firms pay workers their promised wages for the first period and then update their priors using Bayes rule. For the second period, workers are paid their expected productivity. The discount factor between both periods is $\delta=1 / r \in(0,1) .{ }^{15}$ Accordingly, the expected lifetime income of an employee with ability $i$ satisfies the following equation

$$
v(p, \mu \mid r, s)=\frac{1}{1+r}\left(w(\mu)+\frac{E\left[w\left(\mu^{\prime}\right) \mid p, \mu, s\right]}{r}\right),
$$

where $\mu^{\prime}$ denotes the second period belief of the industry and $E[\cdot \mid p, \mu, s]$ is the expectation operator conditional on the industry's prior, worker's type and speed of learning. The scale factor $1 /(1+r)$ ensures that the numerical value of receiving the wage $w$ twice is $w / r$.

As the updated belief $\mu^{\prime}$ is formed according to Bayes rule, we have

$$
\mu^{\prime}(\mu, G)=\frac{\mu d}{\mu d+(1-\mu)(1-d)} \quad \text { and } \quad \mu^{\prime}(\mu, B)=\frac{\mu(1-d)}{\mu(1-d)+(1-\mu) d}
$$

Given these beliefs, second period wages $w(\mu, S)=w\left(\mu^{\prime}(\mu, S)\right)$ depend on the signal $S \in\{B, G\}$ and are given by

$$
w(\mu, G)=\frac{\mu d p_{h}+(1-\mu)(1-d) p_{l}}{\mu d+(1-\mu)(1-d)} \quad \text { and } \quad w(\mu, B)=\frac{\mu(1-d) p_{h}+(1-\mu) d p_{l}}{\mu(1-d)+(1-\mu) d} .
$$

Weighting these two payoffs with their respective probabilities yields the expected wage in the second period

$$
E\left[w\left(\mu^{\prime}\right) \mid p_{l}, \mu\right]=(1-d) w(\mu, G)+d w(\mu, B) \quad \text { and } \quad E\left[w\left(\mu^{\prime}\right) \mid p_{h}, \mu\right]=d w(\mu, G)+(1-d) w(\mu, B) .
$$

Straightforward but cumbersome computations show that $v(p, \mu \mid r, s)$ fulfills all the requirements for strong learning listed in Definition 1 with the single exception that $\lim _{s \rightarrow \infty} v(p, \mu \mid r, s) \neq p / r$. Property P3 does not hold because employer learning matters solely for wages in the second of two fixed periods and so the weight of the starting wage does not become negligible in the limit. This is why the Reports model generates a weak value function in the sense of Definition 1.

\footnotetext{
${ }^{15}$ This notation is introduced for consistency with the discounting factor in Definition 1 and the continuous time model. Alternatively, one can consider that workers are paid their expected productivity over an infinite horizon after the report is generated. In any case, these timing conventions are inessential to the analysis.
} 


\subsection{Discrete time model}

Let us now consider a model where beliefs are explicitly derived from observed output. For simplicity, we maintain the timing conventions of the Reports model so that two periods are sufficient to characterize a worker's career. The only difference is that output is observed by the industry at the end of the first period. Realizations are not deterministic ${ }^{16}$ but randomly drawn from a continuous density $g_{i}(\cdot)$ with a mean equal to the worker's type $p_{i}$ for $i \in\{l, h\}$. The sampling distributions share the same support and are common knowledge. The randomness might be inherent to the production process or to the imperfect precision of the monitoring technology.

Lifetime incomes follow from the same equation (1) as in the Reports model. On the other hand, the updating rule differs as second period beliefs now depend on the output density

$$
\mu^{\prime}(x, \mu)=\frac{\mu g_{h}(x)}{\mu g_{h}(x)+(1-\mu) g_{l}(x)},
$$

whereas wages $w(\mu)=p_{l}+\left(p_{h}-p_{l}\right) \mu$ are given by an affine function of beliefs. In spite of these additional features, the two-period model yields predictions that are similar to that of the more stylized Reports model.

Proposition 1. The value function for the two-period model satisfies property P1 and so exhibits weak learning.

We have not given a specific definition for the speed of learning $s$ in the discrete time model because it would require further parametric restrictions on the $g(\cdot)$ distributions. $^{17}$ In any case, learning is weak in the sense of Definition 1 because P3 does not hold for any conceivable measure of $s$. To see this, consider the limit case where uncertainty becomes negligible as $g_{h}(\cdot)$ and $g_{l}(\cdot)$ converge to Dirac delta functions. Then learning is at its most efficient because types are perfectly revealed at the end of the first period. Nevertheless, the lifetime income of high types converges to $\frac{1}{1+r}\left[w(\mu)+v\left(p_{h}, 1\right)\right]<v\left(p_{h}, 1\right)=p_{h} / r .{ }^{18}$ As in the Reports models, the upper limit property in P3 is not fulfilled because first-period earnings solely depend on the prior and thus do not vary with the speed of learning.

\subsection{Continuous time model}

In the last two models, the length of time required to elicit information is treated as a primitive parameter. This is an artifice of the two-period structure. To the contrary, industries where learning

\footnotetext{
${ }^{16}$ Otherwise learning would be perfect by the end of the first period.

${ }^{17}$ For example, it is shown in next sub-section that when both $g_{i}(\cdot)$ distributions are normal with common variance $\sigma^{2}$, a natural measure for $s$ is the signal/noise ratio: $\left(p_{h}-p_{l}\right) / \sigma^{2}$.

${ }^{18}$ Conversely, the lifetime income of low types converges to $\frac{1}{1+r}\left[w(\mu)+v\left(p_{l}, 0\right)\right]>v\left(p_{l}, 0\right)=p_{l} / r$.
} 
is more efficient should also be characterized by shorter periods of information acquisition. We now address this issue by characterizing Bayesian learning in a continuous time set-up and establishing that it gives rise to strong learning in the sense of Definition 1.

We assume that output realizations are random draws from a Gaussian distribution with a time invariant average productivity. ${ }^{19}$ Thus the cumulative output $X_{t}$ of a match of duration $t$ with a worker of type $i \in\{l, h\}$, follows a Brownian motion with drift

$$
d X_{t}=p_{i} d t+\sigma d Z_{t}
$$

where $d Z_{t}$ is the increment of a standard Brownian motion. The cumulative output $\left\langle X_{t}\right\rangle$ is observed by both parties. The employer uses the filtration $\left\{\mathcal{F}_{t}^{X}\right\}$ generated by the output sample path to revise his belief about $p_{i}$. The variance $\sigma$ is constant across workers for otherwise firms would be able to infer types with arbitrary precision by observing the quadratic variation of $\left\langle X_{t}\right\rangle{ }^{20}$ Starting from a prior $\mu_{0}$ equals to the fraction of high ability workers in the population, the employer applies Bayes rule to update his belief $\mu_{t} \equiv \operatorname{Pr}\left(p=p_{h} \mid \mathcal{F}_{t}^{X}\right)$. His posterior is therefore given by

$$
\mu\left(X_{t}, t \mid \mu_{0}\right)=\frac{\mu_{0} g_{h}\left(X_{t}, t\right)}{\mu_{0} g_{h}\left(X_{t}, t\right)+\left(1-\mu_{0}\right) g_{l}\left(X_{t}, t\right)},
$$

where $g_{i}\left(X_{t}, t\right) \equiv e^{-\frac{\left(X_{t}-p_{i} t\right)^{2}}{2 \sigma^{2} t}}$ is the rescaled ${ }^{21}$ density for a worker of type $i$. The analysis is simplified by the change of variable $\theta_{t} \equiv \mu_{t} /\left(1-\mu_{t}\right)$. $\theta_{t}$ is the ratio of "good" to "bad" belief. Given that $\mu_{t}$ is defined over $] 0,1\left[, \theta_{t}\right.$ takes values over the positive real line. It follows from (2) that

$$
\theta\left(X_{t}, t \mid \theta_{0}\right)=\theta_{0} \frac{g_{h}\left(X_{t}, t\right)}{g_{l}\left(X_{t}, t\right)}=\theta_{0} e^{\frac{s}{\sigma}\left(X_{t}-\frac{1}{2}\left(p_{h}+p_{l}\right) t\right)},
$$

where $s \equiv\left(p_{h}-p_{l}\right) / \sigma$ is the signal/noise ratio of output. On the one hand, a larger productivity difference between types increases the informativeness of the observations. On the other hand, a higher variance hinders the industry's ability to identify the mean of the output distribution. Thus the bigger $s$, the more efficient learning is. By Ito's lemma, the stochastic differential equation satisfied by the belief ratio reads

$$
\begin{aligned}
d \theta\left(X_{t}, t \mid \theta_{0}\right) & =\frac{\partial \theta\left(X_{t}, t \mid \theta_{0}\right)}{\partial X_{t}} d X_{t}+\frac{\partial^{2} \theta\left(X_{t}, t \mid \theta_{0}\right)}{\partial X_{t}^{2}} \frac{\sigma^{2}}{2} d t+\frac{\partial \theta\left(X_{t}, t \mid \theta_{0}\right)}{\partial t} d t \\
& =\theta\left(X_{t}, t \mid \theta_{0}\right)\left(\frac{s}{\sigma}\right)\left[d X_{t}-p_{l} d t\right]
\end{aligned}
$$

\footnotetext{
${ }^{19}$ One can easily verify that letting workers accumulate general human capital would not substantially modify our conclusions.

${ }^{20}$ See, for instance, Chung and Williams (1990).

${ }^{21}$ The factor $[\sigma \sqrt{2 \pi t}]^{-1}$ is omitted because it simplifies in (2).
} 
Replacing in (4) the law of motion of $X_{t}$, i.e. $d X_{t}=p_{i} d t+\sigma d Z_{t}$, yields the following stochastic differential equations

(i) Low ability worker: $d \theta_{t}=\theta_{t} s d Z_{t}$,

(ii) High ability worker : $d \theta_{t}=\theta_{t} s\left(s d t+d Z_{t}\right)$.

The belief ratio $\theta_{t}$ increases with time for high types and follows a martingale for low types. ${ }^{22}$ In both cases, a higher $\sigma$ lowers the volatility of beliefs because larger idiosyncratic shocks hamper signal extraction. We are now in a position to derive expected lifetime incomes as a function of beliefs. Conditional on a given cumulative output $X_{t}$, high and low types earn the same wage. Their expected lifetime incomes differ nonetheless because high types are more optimistic about future prospects. Using the laws of motion above, one can derive the Hamilton-Jacobi-Bellman equations

$$
\begin{aligned}
& r v\left(p_{l}, \theta\right)=w(\theta)+\frac{1}{2}(\theta s)^{2} v^{\prime \prime}\left(p_{l}, \theta\right) \\
& r v\left(p_{h}, \theta\right)=w(\theta)+\theta s^{2} v^{\prime}\left(p_{h}, \theta\right)+\frac{1}{2}(\theta s)^{2} v^{\prime \prime}\left(p_{h}, \theta\right) .
\end{aligned}
$$

Imposing the boundary conditions, $\lim _{\theta \rightarrow 0} v\left(p_{i}, \theta\right)=p_{l} / r$ and $\lim _{\theta \rightarrow \infty} v\left(p_{i}, \theta\right)=p_{h} / r$ for $i \in\{l, h\}$, yields the following closed-form solutions for the two ordinary differential equations. ${ }^{23}$

Proposition 2. For the continuous time model, the expected lifetime incomes of workers as a function of the belief ratio $\theta$ are given by

$$
v\left(p_{l}, \theta\right)=\frac{2 \sigma}{s \Delta}\left(\theta^{\alpha^{-}} \int_{0}^{\theta} \frac{1}{(1+x) x^{\alpha^{-}}} d x+\theta^{\alpha^{+}} \int_{\theta}^{\infty} \frac{1}{(1+x) x^{\alpha^{+}}} d x\right)+\frac{p_{l}}{r}
$$

and

$$
v\left(p_{h}, \theta\right)=\frac{2 \sigma}{s \Delta}\left(\theta^{\gamma^{-}} \int_{0}^{\theta} \frac{1}{(1+x) x^{\gamma^{-}}} d x+\theta^{\gamma^{+}} \int_{\theta}^{\infty} \frac{1}{(1+x) x^{\gamma^{+}}} d x\right)+\frac{p_{l}}{r},
$$

where $\alpha^{+}=\frac{1}{2}(1+\Delta), \alpha^{-}=\frac{1}{2}(1-\Delta), \gamma^{+}=\frac{1}{2}(-1+\Delta), \gamma^{-}=\frac{1}{2}(-1-\Delta)$, and $\Delta=\sqrt{1+8\left(\frac{r}{s^{2}}\right)}$.

The value function satisfies properties P1, P2, and P3, and hence exhibits strong learning.

\footnotetext{
${ }^{22}$ It may be surprising that the belief ratio $\theta_{t}$ does not drift downward when the worker is of the low type. This is because the belief ratio $\theta_{t}$ is a convex function of $\mu_{t}$. Reversing the change of variable shows that, as one might expect, the belief $\mu_{t}$ is a strict supermartingale when the worker's ability is low.

${ }^{23}$ The closed-form solution is of independent interest, and the fact that it can be derived is an additional (technical) contribution of this paper. To the best of our knowledge, the expressions available in the literature are based on the premise that information is symmetric (Moscarini, 2005). In the working paper version of this article we also show how to obtain a solution for cases where senders are uncertain about their productivity. As discussed in the Appendix, the derivation crucially relies on the change of variable from $\mu$ to $\theta$.
} 


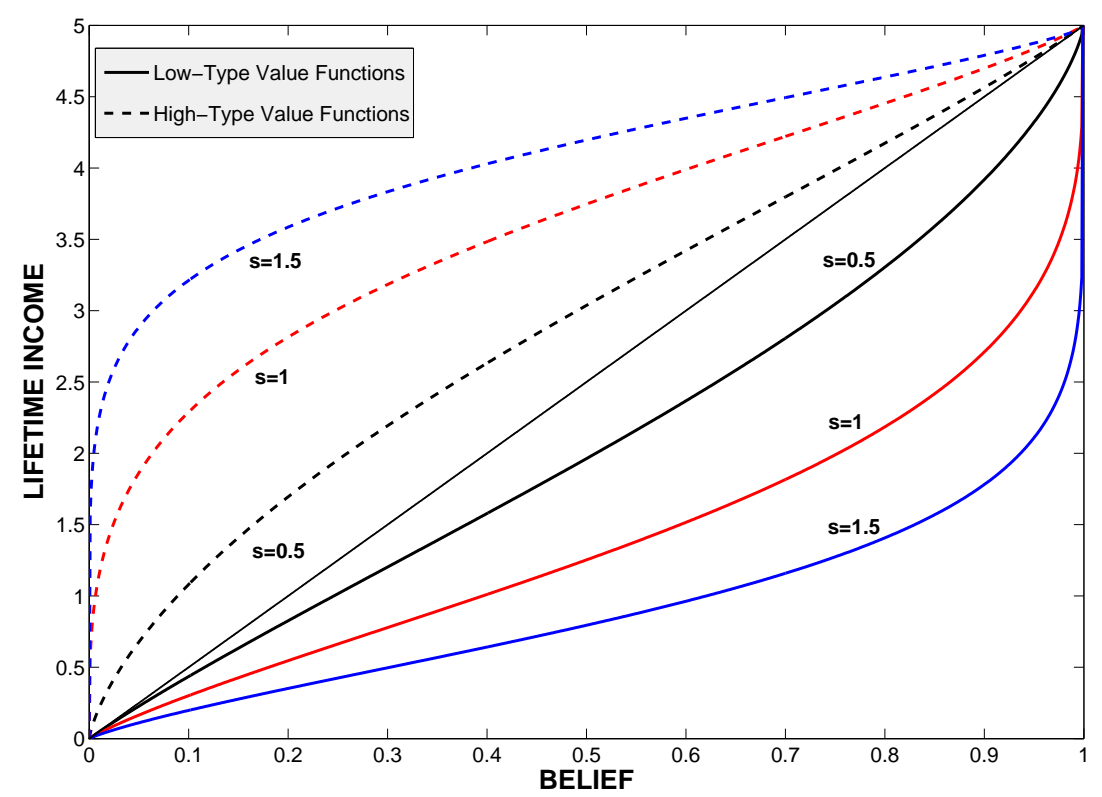

Figure 1: Workers' value functions in the continuous-time model. Parameters: $r=0.2, \mu_{l}=0, \mu_{h}=1$.

The continuous-time model hence provides a computable example of strong learning. Plots of the value functions for a particular numerical example and several values of $s$ are shown in Figure 1, illustrating the properties listed in Definition 1. First, for any given belief $\mu \in(0,1)$, the expected incomes of low and high types are respectively smaller and bigger than the discounted value of their current wage, hence P1 is satisfied. Second, as stated in P2, the gap increases when learning becomes more efficient. Finally, the value functions converge to the discounted value of current wages when $s$ goes to zero and to step functions when $s$ goes to infinity, as required by P3.

\section{Equilibrium Analysis}

Having provided alternative micro foundations for the assumptions laid out in Section 2, we proceed to characterize the signaling equilibria of the game. We start with pure strategy equilibria, which are fully described by the beliefs $\mu(e)$ and the education levels selected by both types, $e_{l}$ and $e_{h}$. If $e_{l} \neq e_{h}$, one speaks of a separating equilibrium, while the equilibrium is called pooling if $e_{l}=e_{h}$. In the second sub-section, we characterize mixed-strategy equilibria. 


\subsection{Pure-strategy equilibria}

Separating equilibria are such that abilities are perfectly revealed: Depending on the education signal, the beliefs of the industry on the equilibrium path are either $\mu=0$ or $\mu=1$. It follows that each type receives an initial wage equal to his productivity and $\mathrm{P} 1$ implies that $v\left(p_{i}, 1\right)=p_{i} / r$ for $i \in\{l, h\}$. In other words, employer learning does not affect separating equilibria.

Proposition 3. Consider any value function. An education profile $\left(e_{l}, e_{h}\right)$ with $e_{l} \neq e_{h}$ can be sustained as a (separating) signaling equilibrium if and only if

(i) $e_{l}=0$, and

(ii) $e_{h} \in\left[\underline{e_{h}}, \overline{e_{h}}\right]$ where $\overline{e_{h}}>\underline{e_{h}}>0$ and these two education levels are uniquely defined by

$$
c\left(p_{l}, \underline{e_{h}}\right)-c\left(p_{l}, 0\right)=\frac{1}{r}\left(p_{h}-p_{l}\right)=c\left(p_{h}, \overline{e_{h}}\right)-c\left(p_{h}, 0\right) .
$$

Accordingly, the set of separating equilibria does not depend on the value function.

In pooling equilibria, both types select the same education level $e_{p}$ and are therefore offered the same initial wage $w\left(\mu_{0}\right)$. Contrary to the model without learning, Bayesian updating leads to different lifetime incomes $v\left(p_{h}, \mu_{0}\right)>v\left(p_{l}, \mu_{0}\right)$. As shown in the following proposition, this implies that the set of pooling equilibria shrinks as the speed of learning increases.

Proposition 4. Consider any value function. A common education level $e_{p}$ can be sustained as a pooling equilibrium if and only if $e_{p} \in\left[0, \overline{e_{p}}\right]$ where $\overline{e_{p}}>0$ is uniquely defined by the equation

$$
c\left(p_{l}, \overline{e_{p}}\right)-c\left(p_{l}, 0\right)=v\left(p_{l}, \mu_{0}\right)-\frac{p_{l}}{r} .
$$

The upper bound fulfills $\overline{e_{p}}<\underline{e_{h}}$, where $\underline{e_{h}}$ is as defined in Proposition 3. Further, under strong learning, $\overline{e_{p}}$ is strictly decreasing in the speed of learning $s$ and $\lim _{s \rightarrow \infty} \overline{e_{p}}=0$.

The set of pooling equilibria is given by a subset of the one in Spence's model. Learning lowers the upper-bound $\overline{e_{p}}$ because the incentives for low types to mimic high types decrease as the ability of firms to detect their productivity increases. Under strong learning and in the limit as $s$ goes to infinity, types are immediately revealed and education is worthless as a signal. This is why the set of pooling equilibria collapses to the minimum level of education. 


\subsection{Mixed-strategy equilibria}

As usual in signaling games, the model admits a plethora of mixed-strategy equilibria. Those are often referred to as partial pooling or hybrid equilibria. The following Proposition shows that learning supports mixed-strategy equilibria which are qualitatively different from those possible in Spence's model. ${ }^{24}$

Proposition 5. Consider any value function. For any mixed-strategy signaling equilibrium, there exists a set of education levels $E_{p}$ such that the following properties hold:

(i) The support of the low types' strategy consists of $E_{p}$ and at most a further education level, $e_{l}=0$, such that $e_{l}<e$ for all $e \in E_{p}$.

(ii) The support of the high types' strategy consists of $E_{p}$ and at most a further education level $e_{h}$, such that $e_{h}>$ e for all $e \in E_{p}$.

(iii) The industry's beliefs $\mu$ are strictly increasing on $E_{p}$.

In Spence's model, $E_{p}$ contains at most one education level, but this is not true for general value functions.

Equilibria with $E_{p}=\emptyset$ are the separating equilibria of Proposition 3. The pooling equilibria of Proposition 4 are such that $E_{p}=\left\{e_{p}\right\}$ where no additional education levels are chosen. In Spence's model, the set $E_{p}$ (if nonempty) always consist of a unique education level, but this is not true in general for other value functions. Employer learning qualitatively enriches the set of mixed equilibria: multiple pooling may arise, i.e. equilibria with more than one education level chosen by both types. Below we construct an illustrative example where low types randomize among three education levels, two of which are also chosen by high types. This is the simplest situation which is not possible as an equilibrium of Spence's model. More complex examples are of course conceivable, e.g. with continuous common support or multiple education levels, all of them chosen by both types.

Notice that, although the set of mixed-strategy equilibria might be large and capture complex phenomena as multiple pooling, such equilibria are far from being arbitrary. In particular, the monotonicity property (iii) above shows that there can be no counterintuitive effects. Education remains

\footnotetext{
${ }^{24}$ Proposition 5 can be refined assuming that, for all $\mu \in(0,1), \partial^{2} v\left(p_{h}, \mu\right) / \partial \mu^{2}<\partial^{2} v\left(p_{l}, \mu\right) / \partial \mu^{2}$, a property that can easily be established in the reports and discrete time models (and is also illustrated in Figure 1). Then, $P 1$ implies that there exists a unique belief $\mu^{*} \in(0,1)$ such that $\partial v\left(p_{l}, \mu^{*}\right) / \partial \mu \lesseqgtr \partial v\left(p_{h}, \mu^{*}\right) / \partial \mu$ if and only if $\mu \lesseqgtr \mu^{*}$. It is then straightforward to show that, under weak or strong learning, $\mu(e)>\mu^{*}$ for all $e \in E_{p}$ except possibly the minimum education level in $E_{p}$ (if a minimum exists).
} 
informative in the sense that a higher (equilibrium) education level is always associated to a higher belief that the worker is of the high type.

Before presenting our multiple-pooling example, let us briefly discuss the intuition for this result. Consider first the model without employer learning. The basic argument for the impossibility of multiple pooling is as follows. If a given type chooses two different levels of education with positive probability, then he must be indifferent between those two education levels, implying that the difference in expected income must equal that in educational costs. Without employer learning, each level of education results in identical incomes for both types. By the single crossing property, however, differences in educational costs across signals are higher for low types. Hence both types cannot randomize over the same set. The contradiction disappears with employer learning because, even when priors are identical, lifetime earnings differ across types.

An example with multiple pooling. We base our example on the Reports model described before. First, we specify numerical values for the parameters in order to simplify the computations. Let the detection probability be $d=3 / 4$, the productivities $p_{l}=1$ and $p_{h}=2$, the discount parameter $r=11 / 10$, and the cost function be given by $c(p, e)=\frac{9 e}{8+p}$. The exogenously given population share of high types is $\mu_{0}=1 / 2$.

The equilibrium is as follows. Three signals are chosen $e_{0}=0, e_{1}$, and $e_{2}$, where only low productivity workers send $e_{0}=0$, but both types choose the other two levels with positive probability. Table 1 specifies their numerical values, the probability $q(e \mid p)$ with which each type selects them, and the corresponding industry beliefs derived from Bayes' rule. Note how equilibrium beliefs are increasing in education as predicted by part (iii) of the last proposition.

\begin{tabular}{|c|c|c|c|}
\hline & $e_{0}$ & $e_{1}$ & $e_{2}$ \\
\hline$e$ & 0 & $\frac{185}{462} \cong 0.40$ & $\frac{95}{154} \cong 0.62$ \\
\hline$q\left(e \mid p_{l}\right)$ & $\frac{4}{9}$ & $\frac{1}{3}$ & $\frac{2}{9}$ \\
\hline$q\left(e \mid p_{h}\right)$ & 0 & $\frac{1}{3}$ & $\frac{2}{3}$ \\
\hline$\mu(e)$ & 0 & $\frac{1}{2}$ & $\frac{3}{4}$ \\
\hline
\end{tabular}

Table 1: An equilibrium with multiple pooling. 
Given these beliefs, we have

$$
v\left(p_{l}, \mu\left(e_{2}\right)\right)-e_{2}=v\left(p_{l}, \mu\left(e_{1}\right)\right)-e_{1}=v\left(p_{l}, 0\right)=\frac{10}{11},
$$

and

$$
v\left(p_{h}, \mu\left(e_{1}\right)\right)-\frac{9}{10} e_{1}=v\left(p_{h}, \mu\left(e_{2}\right)\right)-\frac{9}{10} e_{2}=\frac{977}{924} \cong 1.0574>\frac{10}{11} .
$$

Thus each type is indifferent among the specified education levels in the support of their own strategies. We still have to establish that no type has an incentive to deviate. This is done specifying the out-ofequilibrium beliefs $\mu(e)=0$ for all $e \notin\left\{e_{0}, e_{1}, e_{2}\right\}$. Under these beliefs, any such education level leads to a lifetime earning of $10 / 11$ and strictly positive education costs, thus neither of the two types has an incentive to undertake those deviations.

Last, we must consider whether high types have an incentive to deviate to $e_{0}$. This deviation results in a payoff of 10/11, which is strictly smaller than the high types' payoffs with $e_{1}$ and $e_{2}$. Hence, the postulated profile is a signaling equilibrium with multiple pooling, a qualitatively new phenomenon which could not occur in Spence's model.

\section{The Intuitive Criterion}

In order to narrow the large set of equilibria typically found in signaling games, a number of refinement concepts have been developed (especially for games with three or more types). The Intuitive Criterion (Cho and Kreps 1987), however, remains a milestone in the analysis of signaling games. When there are only two types, it is well known that the Intuitive Criterion confers a predictive power to Spence's model by ruling out all but one separating equilibrium, known as the Riley equilibrium. The purpose of this section is to show that this does not hold true when beliefs can be updated after the signaling stage. More precisely, we prove that, even though the Riley equilibrium retains its importance as the only separating equilibrium fulfilling the Intuitive Criterion, it is in general not true that all pooling (or mixed) equilibria are ruled out.

A signaling equilibrium is said to fail the Intuitive Criterion if some type could strictly profit by sending a non-equilibrium signal, provided that the sender adopts non-equilibrium beliefs satisfying the following requirement: Assign probability zero to types which could never conceivably profit by sending the considered signal. In our set-up, the Intuitive Criterion amounts to the following. Fix a signaling equilibrium. Say that an unused signal $e$ is equilibrium-dominated for type $p$ if the equilibrium payoff of type $p$ is strictly larger than the payoff that type $p$ would receive with signal $e$, given any conceivable (non-equilibrium) belief of the industry $\mu^{\prime}(e)$. The equilibrium is said to fail the Intuitive Criterion if there exists a signal $e$ and a type $p$ such that the equilibrium payoff of type 
$p$ is strictly smaller than the minimum payoff that this type could get by sending signal $e$, given any possible (non-equilibrium) beliefs $\mu^{\prime}(e)$ of the industry which concentrate on the set of types for which signal $e$ is not equilibrium dominated.

Separating equilibria. In the absence of employer learning, the Riley equilibrium is the only separating equilibrium which survives the Intuitive Criterion: Low types do not acquire any education and high types send the signal $\underline{e_{h}}$ defined in Proposition 3, implying that low types are indifferent between their equilibrium strategy and acquiring education $e_{h}$ in order to receive $v\left(p_{l}, 1\right)=p_{h} / r$. Given that separating equilibria do not depend on the value function, it is not surprising that the result carries over to our set-up. ${ }^{25}$

Proposition 6. Consider any value function. The only separating equilibrium which survives the Intuitive Criterion is the Riley equilibrium.

Survival of pooling equilibria. We now focus on pooling equilibria and use $e_{p}$ to denote the common level of education. First, notice that no signal $e<e_{p}$ can be equilibrium dominated for either type. Further, if a deviation to $e>e_{p}$ is equilibrium-dominated for the high type, it is never profitable for the low type (in the sense of the Intuitive Criterion) to choose $e$. For such a deviation would induce industry's beliefs $\mu(e)=0$ and low types would obtain lower payoffs $\left(p_{l} / r\right)$ than at the pooling equilibrium but incur strictly larger educational costs.

Hence, a pooling equilibrium with education level $e_{p}$ fails the Intuitive Criterion if and only if there exists a signal $e>e_{p}$ such that it is equilibrium dominated for the low types but would result in a better payoff than in equilibrium for the high types when the industry places zero probability on the event that the sender is of the low type given signal $e$, i.e.

$$
\begin{aligned}
& \frac{p_{h}}{r}-c\left(p_{l}, e\right)<v\left(p_{l}, \mu_{0}\right)-c\left(p_{l}, e_{p}\right) \quad \text { (equilibrium dominance for the low type), } \\
& \frac{p_{h}}{r}-c\left(p_{h}, e\right)>v\left(p_{h}, \mu_{0}\right)-c\left(p_{h}, e_{p}\right) \quad \text { (profitable deviation for the high type). }
\end{aligned}
$$

The equilibrium dominance condition (ED) implies that, even in the best-case scenario where the worker could forever deceive employers, deviating to $e$ is not attractive to low types. The firm can therefore infer by forward induction that any worker with an off-equilibrium signal $e$ has a high productivity. The "profitable deviation" condition (PD) implies in turn that credibly deviating to $e$ is indeed profitable for the high type. Thus such an $e_{p}$ fails the Intuitive Criterion, or, following the terminology of Kohlberg and Mertens (1986), is not stable.

\footnotetext{
${ }^{25}$ The proof of Proposition 6 is not included in the Appendix because it follows from standard arguments.
} 
Let $e^{*}\left(e_{p}\right)$ denote the minimum education level that does not trigger a profitable deviation for low ability workers, so that (ED) holds with equality at $e^{*}\left(e_{p}\right)$. Condition (ED) can then be rewritten as $e>e^{*}\left(e_{p}\right)$. Analogously, let $e^{* *}\left(e_{p}\right)$ be such that (PD) holds with equality, then (PD) can be rewritten as $e<e^{* *}\left(e_{p}\right)$. These two thresholds always exist because $c(p, e)$ is continuous and strictly increasing in $e .{ }^{26}$ There exists an education level satisfying both conditions (ED) and (PD) if and only if $e^{*}\left(e_{p}\right)<e^{* *}\left(e_{p}\right)$. This condition provides us with a straightforward proof that all pooling equilibria fail the Intuitive Criterion in the model without learning. Assume that $e_{p}$ is stable, so that condition (PD) is not satisfied at $e^{*}\left(e_{p}\right)$. This can be true if and only if

$$
v\left(p_{h}, \mu_{0}\right)-v\left(p_{l}, \mu_{0}\right) \geq c\left(p_{l}, e^{*}\left(e_{p}\right)\right)-c\left(p_{l}, e_{p}\right)-\left[c\left(p_{h}, e^{*}\left(e_{p}\right)\right)-c\left(p_{h}, e_{p}\right)\right] .
$$

In Spence's model or as $s \rightarrow 0$, we have $v\left(p_{l}, \mu_{0}\right)=v\left(p_{h}, \mu_{0}\right)=w\left(\mu_{0}\right) / r$. The left hand side of inequality (5) converges to zero while the right-hand side is strictly positive by the single crossing property. The contradiction illustrates that, in the basic signaling model, one can always find a credible and profitable deviation for high types.

When workers' abilities are also revealed on-the-job, the premise leading to a contradiction is no longer true. As stated in property P1, the expectations of high types are higher than those of low types. In other words, $v\left(p_{h}, \mu_{0} \mid r, s\right)>v\left(p_{l}, \mu_{0} \mid r, s\right)$ for all $s>0$, and so inequality (5) can hold true for some parameter configurations. We summarize these observations in the following result.

Proposition 7. The Intuitive Criterion rules out all pooling equilibria in the absence of learning, but this is no longer true for value functions with either weak or strong learning.

In order to complete the proof of the statement, we need to exhibit an example where some pooling equilibria survive the Intuitive Criterion. For the sake of computability, we will use the Reports model again. We keep the same parameter values as in the previous example. ${ }^{27}$ Applying Proposition 4 yields that pooling equilibria correspond to education levels $e_{p} \in\left[0, \overline{e_{p}}\right]$ where $\overline{e_{p}}>0$ is defined by

$$
\overline{e_{p}}=v\left(p_{l}, \mu_{0}\right)-\frac{p_{l}}{r}=\frac{185}{462} \cong 0.40 \text {. }
$$

We claim that all pooling equilibria survive the Intuitive Criterion. To see this, we compute $e^{*}$ and $e^{* *}$ as discussed before. Taking equalities in conditions (ED) and (PD) we obtain

$$
e^{*}\left(e_{p}\right)=\frac{p_{h}}{r}-v\left(p_{l}, \mu_{0}\right)+e_{p}=\frac{235}{462}+e_{p} \cong 0.51+e_{p}
$$

\footnotetext{
${ }^{26}$ To see this, observe first that, because $v\left(p_{l}, \mu_{0}\right)<p_{h} / r$, we have $p_{h} / r-c\left(p_{h}, e_{p}\right)>v\left(p_{h}, \mu_{0}\right)-c\left(p_{h}, e\right)$ for $e=e_{p}$. Further, the cost function $c(p, e)$ is continuous, strictly increasing, and convex in $e$, hence the inequality is reversed for $e$ large enough. This implies that $e^{*}\left(e_{p}\right)$ and $e^{* *}\left(e_{p}\right)$ are well-defined. It also follows that $e^{*}\left(e_{p}\right)>e_{p}$ and $e^{* *}\left(e_{p}\right)>e_{p}$. ${ }^{27}$ That is: $d=3 / 4, r=11 / 10, p_{l}=1, p_{h}=2, \mu_{0}=1 / 2$, and $c(p, e)=\frac{9 e}{8+p}$.
} 
and

$$
e^{* *}\left(e_{p}\right)=\frac{10}{9}\left(\frac{p_{h}}{r}-v\left(p_{h}, \mu_{0}\right)\right)+e_{p}=\frac{925}{2079}+e_{p} \cong 0.44+e_{p}
$$

i.e. $e^{* *}\left(e_{p}\right)<e^{*}\left(e_{p}\right)$ for all $e_{p}$, which yields the desired conclusion.

According to property P2, the faster learning is, the wider the gap in expected income between low and high types. This suggests that a pooling equilibrium is more likely to be stable when signal extraction is efficient. The following proposition substantiates this intuition, showing that any pooling equilibrium is stable for a high enough speed of learning.

Proposition 8. Consider a value function with strong learning. Then, for any education level $e_{p}$, there exists a speed of learning $s^{*}\left(e_{p}\right)$ such that, for any $s \geq s^{*}\left(e_{p}\right)$, if $e_{p}$ can be sustained as a pooling equilibrium then it survives the Intuitive Criterion.

Figure 2 illustrates the mechanism behind Propositions 7 and 8. It displays the indifference curves of high and low types when $s=s^{*}\left(e_{p}\right)$ and when $s=0$. The dotted curves correspond to the former case, the undotted curves to the latter one, that is, Spence's model. The level of education $e^{*}\left(e_{p} \mid s\right)$ where condition (ED) holds with equality is given by the point where the indifference curve of low types crosses the horizontal line with intercept $p_{h} / r$. Similarly, the level of education $e^{* *}\left(e_{p} \mid s\right)$ where condition (PD) holds with equality is given by the point where the indifference curve of high types crosses the same horizontal line. The pooling equilibrium $e_{p}$ fails the Intuitive Criterion if and only if $e^{*}\left(e_{p}\right)<e^{* *}\left(e_{p}\right)$. We can therefore conclude that $e_{p}$ is not stable when the indifference curve of low types intersects the horizontal line with intercept $p_{h} / r$ before the indifference curve of high types .

Consider first the basic model without learning. At the pooling level of education $e_{p}$, the two types enjoy the same asset value $w\left(\mu_{0}\right) / r$. The single-crossing property implies that $e^{*}\left(e_{p}\right)$ lies to the left of $e^{* *}\left(e_{p}\right)$, as shown in Figure 2. Thus any pooling equilibrium fails the Intuitive Criterion when there is no learning. Consider now what happens when the speed of learning $s$ increases. As high types are more quickly recognized, their asset value increases and their indifference curve shifts up. Conversely, the indifference curve of low types shifts down. These opposite adjustments shrink the gap between $e^{*}\left(e_{p} \mid s\right)$ and $e^{* *}\left(e_{p} \mid s\right)$. The threshold speed of learning $s^{*}\left(e_{p}\right)$ is identified by the point where the gap vanishes as the two indifference curves concurrently cross the horizontal line with intercept $p_{h} / r$. For any value function, property $\mathrm{P} 3$ ensures that one can always find such a point for any given $e_{p}$ because $\lim _{s \rightarrow \infty} v\left(p_{h}, \mu \mid r, s\right)=p_{h} / r$. Figure 2 also illustrates the fact that $s^{*}\left(e_{p}\right)$ is unique.

The economics behind Proposition 8 makes intuitive sense. When learning is fast, firms easily infer the actual type of their employees. Then the benefits derived from ex-ante signaling are not important. Conversely, when learning is slow, firms learn little from observed outputs. This leaves fewer opportunities for high types to reveal their ability after the signaling stage and thus raises their 


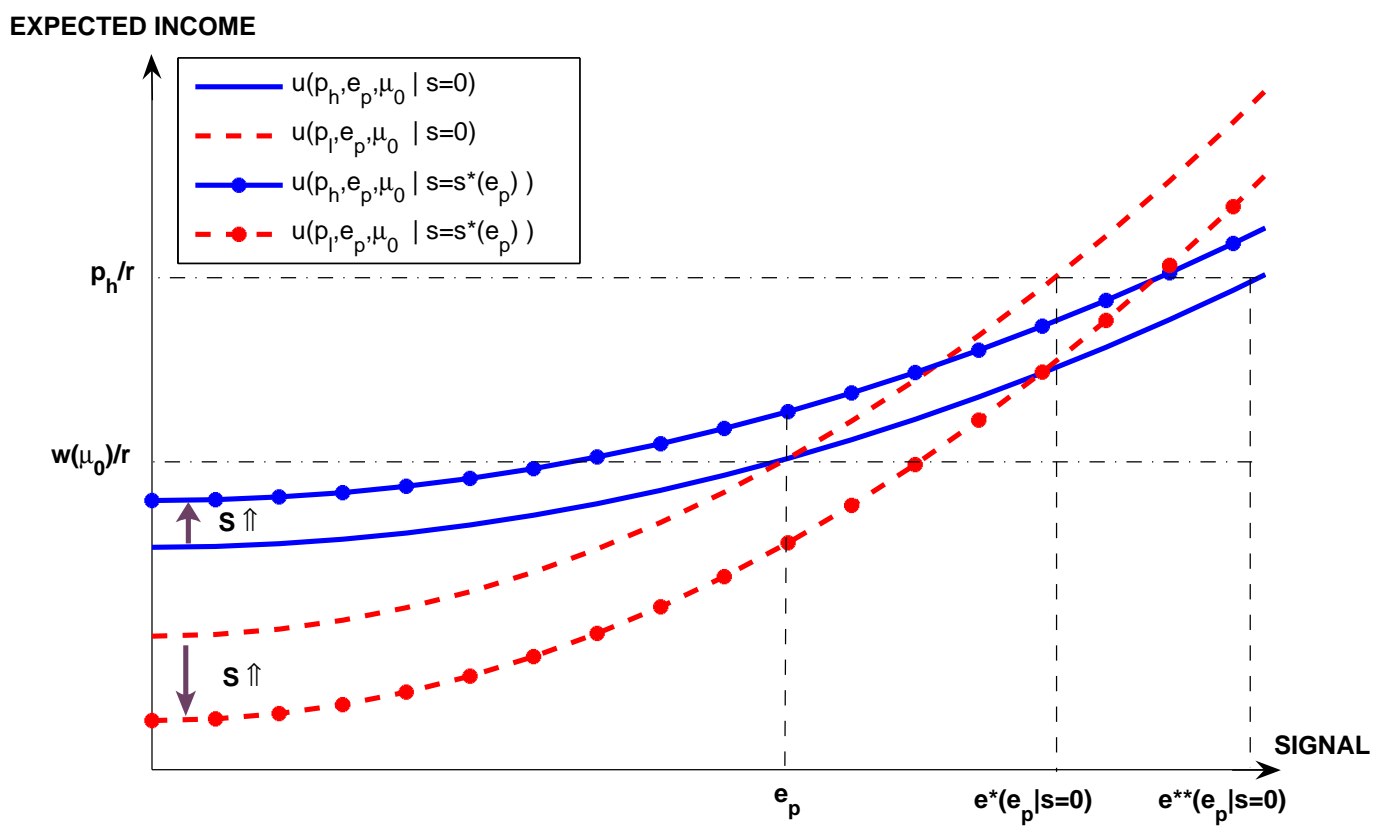

Figure 2: Workers' indifference curves.

incentives to send a message. In the extreme case where the speed of learning goes to zero, all the relevant information is collected prior to labor market entry.

According to Proposition 8, when the speed of learning is high enough, the Intuitive Criterion does not rule out any pooling equilibrium. Proposition 4, however, states that the set of pooling equilibria shrinks as the speed of learning increases. Taken together, these results imply that there are three possibilities: (i) if learning is slow, there is a large set of pooling equilibria, almost all (or all) of which fail the Intuitive Criterion; (ii) if learning is fast, pooling equilibria would survive the Intuitive Criterion, but the set of such equilibria is small; and (iii) for intermediate values of the speed of learning, there exists a sizeable set of pooling equilibria which survive the Intuitive Criterion.

This qualitative classification can be made more precise by assuming that the log of the derivative of $c(p, e)$ with respect to $e$ has (strictly) decreasing differences in $(p, e)$ or, in other words, that $c_{e}(p, e)$ is (strictly) log-submodular: ${ }^{28}$

$$
\frac{c_{e}\left(p_{h}, e^{\prime \prime}\right)}{c_{e}\left(p_{h}, e^{\prime}\right)}>\frac{c_{e}\left(p_{l}, e^{\prime \prime}\right)}{c_{e}\left(p_{l}, e^{\prime}\right)}, \quad \text { whenever } e^{\prime \prime}>e^{\prime}
$$

\footnotetext{
28 This assumption might seem restrictive but it is actually satisfied by the functions commonly used to illustrate the single crossing property. Textbook examples of cost functions usually exhibit log-linear marginal costs which, as stated at the end of Proposition 9, yields an even simpler division of the parameter space.
} 
Proposition 9. Let $c_{e}$ be strictly log-submodular, and consider any value function with strong learning. Let $\overline{e_{p}}(s)$ be as in Proposition 4. Then, there exists an $s^{*}(0)>0$ and an $\bar{s}>s^{*}(0)$ such that

(a) For all $s \in\left[0, s^{*}(0)[\right.$, all pooling equilibria fail the Intuitive Criterion.

(b) For all $s \in\left[s^{*}(0), \bar{s}\left[\right.\right.$, there exists $\widetilde{e}(s)$, strictly decreasing in $s$, such that (i) $\widetilde{e}(s)<\overline{e_{p}}(s)$, (ii) all pooling equilibria with education level $e_{p} \in[0, \widetilde{e}(s)]$ survive the Intuitive Criterion, and (iii) all pooling equilibria with education level $\left.\left.e_{p} \in\right] \widetilde{e}(s), \overline{e_{p}}(s)\right]$ fail the Intuitive Criterion.

(c) For all $s \geq \bar{s}$, all pooling equilibria survive the Intuitive Criterion.

If $c_{e}$ is log-linear, the result holds with $s^{*}(0)=\bar{s}$, i.e. case (b) cannot occur.

This result is illustrated in Figure 3. Whereas the statement in Proposition 8 is local, assuming log-submodularity allows for a global characterization of the region where the Intuitive Criterion bites. Log-submodularity is more stringent than the single crossing property because it implies that marginal educational costs diverge. If that property were not satisfied, an increase in the level of education could restore the stability of some pooling equilibria. Hence, when $c_{e}(p, e)$ is not log-submodular, equilibrium stability does not always divide the $(s, e)$ space into two non-overlapping regions.

A comment on mixed-strategy equilibria. In Spence's model, all mixed-strategy equilibria fail the Intuitive Criterion. Essentially, the reason is that any such equilibrium involves one (and, in Spence's model, only one) pooling education level on which an argument analogous to that used to discard pooling equilibria can be based.

In our case, it is not difficult to see that the same argument which shows that pooling equilibria might survive the Intuitive Criterion will also allow some mixed equilibria to be stable. For instance, suppose low types do not randomize at all. In such an equilibrum, solely two education levels are used in equilibrium: A "pooling" one $\left(e_{p}\right)$, and a higher $\left(e_{h}>e_{p}\right)$ chosen only by high types. Such equilibria will behave similarly to pooling ones, e.g. if $e_{h}$ is relatively close to $e_{p}$.

However, it is also possible to show that more complex mixed-strategy equilibria might survive the Intuitive Criterion. Consider a mixed strategy equilibrium with nonempty set of pooling education levels $E_{p}$, and suppose, for the sake of simplicity, that this equilibrium also involves an education level $e_{l}=0$ being chosen by low types only. This implies that the equilibrium payoff of low types is simply $p_{l} / r-c\left(p_{l}, 0\right)$. As in Proposition 6 , any $e>\underline{e_{h}}$ is equilibrium-dominated for the workers of low productivity. This also implies that no such equilibrium level might be chosen by the low types in equilibrium, i.e. $e_{p}<\underline{e_{h}}$ for all $e_{p} \in E_{p}$. 


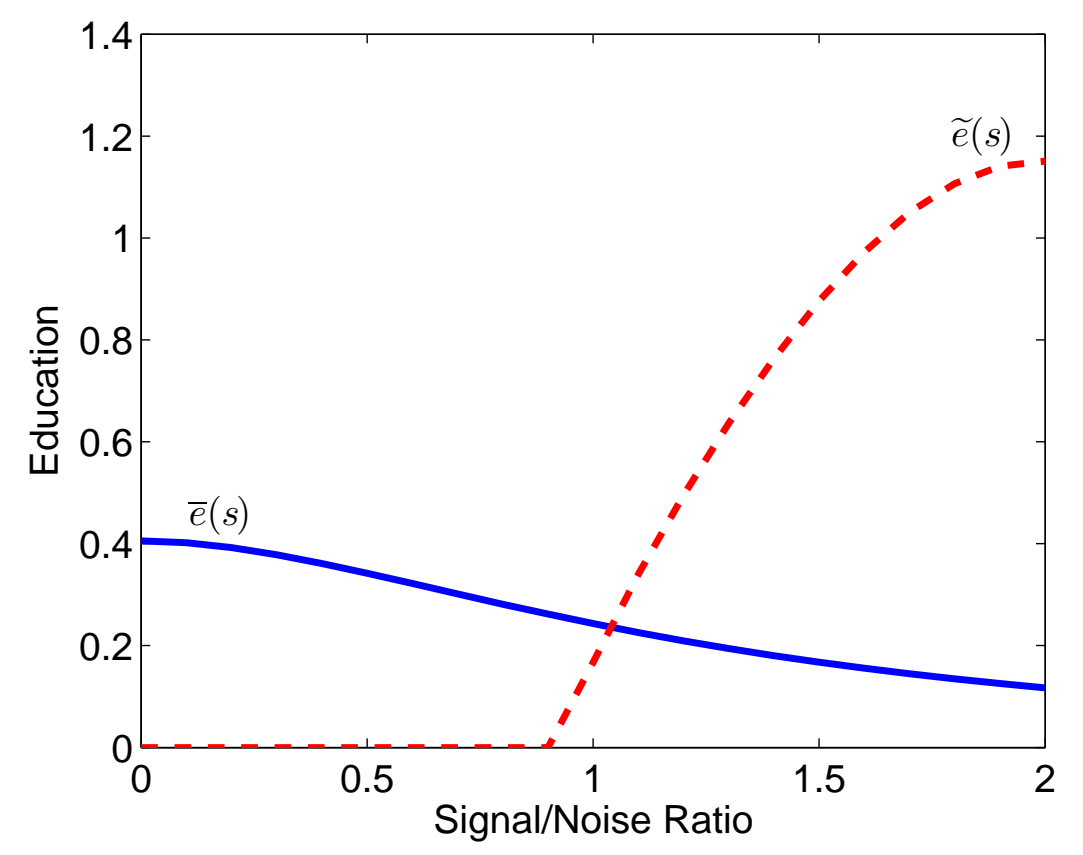

Figure 3: Graphical interpretation of Proposition 9. A pooling equilibrium with education level $e$ exists if and only if $e \leq \bar{e}(s)$. It satisfies the Intuitive Criterion if and only if $e \leq \widetilde{e}(s)$. Parameters: $r=0.2, p_{l}=0.5, p_{h}=1, \mu_{0}=0.5$ and $c(p, e)=\exp (e / p)-1$.

Fix some $e_{p} \in E_{p}$, and let $\mu=\mu\left(e_{p}\right)$. By indifference of the low types we obtain

$$
\frac{p_{l}}{r}-c\left(p_{l}, 0\right)=v\left(p_{l}, \mu\right)-c\left(p_{l}, e_{p}\right),
$$

and the equilibrium payoff of the high types is $v\left(p_{h}, \mu\right)-c\left(p_{h}, e_{p}\right)$. Suppose, for the sake of the argument, that a choice of the Riley education level $\underline{e}_{h}$ would result in the industry setting beliefs of $\mu\left(\underline{e_{h}}\right)=1$. Then, the high types would obtain payoff

$$
\begin{aligned}
\frac{p_{h}}{r}-c\left(p_{h}, \underline{e_{h}}\right) & >\frac{p_{h}}{r}-\left(c\left(p_{l}, \underline{e_{h}}\right)-c\left(p_{l}, e_{p}\right)\right)-c\left(p_{h}, e_{p}\right) \\
& =\frac{p_{h}}{r}-\frac{p_{l}}{r}+v\left(p_{l}, \mu\right)+c\left(p_{l}, 0\right)-c\left(p_{l}, \underline{e_{h}}\right)-c\left(p_{h}, e_{p}\right)=v\left(p_{l}, \mu\right)-c\left(p_{h}, e_{p}\right),
\end{aligned}
$$

where the first inequality follows from the single crossing property, the second one from the low types' indifference condition above, and the last one from the definition of $\underline{e_{h}}$ in Proposition 3. For the model without learning, $v\left(p_{l}, \mu\right)=v\left(p_{h}, \mu\right)$ and one can conclude that education levels close to but higher than the Riley one are equilibrium-dominated for low types and result in a strict gain for high types, thereby leading to a failure of the Intuitive Criterion. This is not true anymore with strong learning, 
because $v\left(p_{l}, \mu\right)<v\left(p_{h}, \mu\right)$. For particular value functions and particular parameter values, also such equilibria might survive the Intuitive Criterion.

It might even be the case that equilibria with multiple pooling (i.e. where both types randomize among at least two different education levels, i.e. $E_{p}$ contains at least two elements) survive the Intuitive Criterion. To see this, consider the multiple pooling equilibrium exhibited in Section 4.2, where low types randomize among $e_{0}=0$ and two positive education levels $e_{1}$ and $e_{2}>e_{1}$, while high types randomize among $e_{1}$ and $e_{2}$. As in the case of pooling equilibria, it is easy to see that such an equilibrium fails the Intuitive Criterion if and only if there exists an education level $e>e_{2}$ which is equilibrium-dominated for the low types but would be strictly profitable for high types if industry reacts with beliefs $\mu(e)=1 .{ }^{29}$ For this example, equilibrium dominance for the low types reduces to

$$
\frac{10}{11}=\frac{p_{l}}{r}-c\left(p_{l}, 0\right)>\frac{p_{h}}{r}-c\left(p_{l}, e\right)=\frac{20}{11}-e,
$$

and a deviation to $e$ would be strictly profitable for the high types if and only if

$$
1.0574 \cong \frac{977}{924}=v\left(p_{h}, \mu\left(e_{2}\right)\right)-c\left(p_{h}, e_{2}\right)<\frac{p_{h}}{r}-c\left(p_{h}, e\right)=\frac{20}{11}-\frac{9}{10} e .
$$

The first condition yields $e>\frac{10}{11}$ and the second reduces to $e<\frac{10}{9}\left[\frac{20}{11}-\frac{977}{924}\right] \cong 0.8453$. Since both are incompatible, it follows that this multiple pooling equilibrium survives the Intuitive Criterion.

Further Equilibrium Refinements. We have focused on the Intuitive Criterion because of its relevance for Spence's model. One could also investigate the predictive power of more sophisticated refinements. First, it is straightforward to check that, even with employer learning, our game is a monotonic signaling game in the sense of Cho and Sobel (1990). Hence, by Proposition 3.1 in that paper, the three refinements that naturally come to mind beyond the Intuitive Criterion actually coincide: Universal Divinity (Banks and Sobel 1987), Never a Weak Best Response (NWBR; Kohlberg and Mertens 1986), and criterion D1 (Cho and Kreps 1987).

Second, it is a relatively simple matter to build (cumbersome) examples showing that, for general cost and value functions, those criteria do not necessarily refine the set of equilibria. Rather than embarking in long computations, we will outline the essential reasoning here. For monotonic signaling games, Cho and Sobel identified a series of conditions which guarantee that the three criteria mentioned above lead to a unique equilibrium (See Proposition 4.4 in Cho and Sobel, 1990). ${ }^{30}$ The only nontechnical condition is A4: If $p<p^{\prime}$ and $e<e^{\prime}$, then $u(e, \mu \mid p) \leq u\left(e^{\prime}, \mu^{\prime} \mid p\right) \Rightarrow u\left(e, \mu \mid p^{\prime}\right)<u\left(e^{\prime}, \mu^{\prime} \mid p^{\prime}\right)$.

\footnotetext{
${ }^{29}$ No education level lower than $e_{2}$ can be equilibrium-dominated for either type. Further, if an education level $e>e_{2}$ would be equilibrium-dominated for the high types, it would never pay for the low type to deviate to it.

${ }^{30}$ Esö and Schummer's (2008) Vulnerability to Credible Deviations provides an alternative interpretation of this selection result within the context of monotonic signaling games
} 
In Cho and Sobel's words, "A4 is crucial to our analysis. It states that if two signal-action pairs yield the same utility to some type of Sender, and one signal is greater (componentwise) than the other, then all higher types prefer to send the greater signal." ${ }^{31}$ In Spence's model, this condition is implied by the single-crossing property, for the wage given an education level is independent of the type. With employer learning, the link fails because lifetime earnings differ across types. Indeed, it is apparent that A4, applied to a job-market signaling model, actually excludes employer learning from the model. Hence there is no reason to expect refinements like D1, Universal Divinity, or NWBR to generically restore equilibrium uniqueness in the absence of strong additional restrictions. ${ }^{32}$

There exists a connection between our results and a different kind of equilibrium refinement criteria. $^{33}$ Mailath, Okuno-Fujiwara, and Postlewaite (1993) postulate the Undefeated Criterion as an alternative to the Intuitive Criterion and argue in favor of pooling equilibria when they Paretodominate the Riley equilibrium. The key argument is that, for some parameter constellations, the pooling equilibrium with zero education level might Pareto-dominate the Riley equilibrium. ${ }^{34}$

There is an interesting connection between our results and Pareto dominance as captured by the Undefeated Criterion. Consider the pooling equilibrium at $e=0$. By definition of the Riley equilibrium education level $\underline{e_{h}}$, the low type is indifferent between receiving the wage $p_{h}$ and sending $\underline{e_{h}}$ and receiving the wage $p_{l}$ at an education level of zero. The payoff at the pooling equilibrium with $e=0$ is strictly larger than the latter, and hence the Riley signal $e_{h}$ is always equilibrium-dominated for the low type. If any pooling equilibrium survives the Intuitive Criterion, then so does the pooling equilibrium with $e=0$. Thus the payoff of the high types at the pooling equilibrium with $e=0$ must be weakly better than at the Riley equilibrium (else the former would fail the Intuitive Criterion). The payoff of the low types is always strictly better. It follows that the pooling equilibrium with $e=0$

\footnotetext{
${ }^{31}$ Note that condition A4 excludes from the onset equilibria with multiple pooling.

${ }^{32}$ Daley and Green (2007) use the D1 criterion to analyze a signaling game with grades, which is closely related to our reports model. Although they rely on a more demanding notion of equilibrium stability, they still need to impose additional restrictions in order to ensure equilibrium uniqueness. Hence, their analysis concurs with ours in concluding that forward induction is vulnerable to the introduction of correlated information after the signaling stage.

${ }^{33}$ We thank an anonymous referee for pointing us in this direction.

${ }^{34}$ The Undefeated Criterion is more involved than Pareto-dominance. Mailath, Okuno-Fujiwara, and Postlewaite (1993) restrict attention to pure-strategy signaling equilibria. Within this class, an equilibrium $\left(\sigma^{\prime}, \mu^{\prime}\right)$ is defeated if a new equilibrium $(\sigma, \mu)$ can be built where a previously unused signal $m$ is used by some types, in such a way that (i) the new equilibrium is a Pareto-improvement for those types (all of them being weakly better off, and at least one being strictly better off), and (ii) for some type using $m$ in $(\sigma, \mu)$, the receiver's out-of-equilibrium belief in $\left(\sigma^{\prime}, \mu^{\prime}\right)$ that the sender is of that type on seeing $m$ can not be explained through conditioning on the set of sender types who do use $m$ in $(\sigma, \mu)$, even allowing for the possibility that indifferent types might have randomized. In the context of Spence's model, for certain parameter values the Riley equilibrium is defeated by pooling equilibria with education levels close to zero, while pooling at zero is undefeated. For other parameter values, the Riley equilibrium is undefeated.
} 
Pareto-dominates the Riley equilibrium. Repeating the argument for pooling equilibria with $e$ close to zero, it can be shown that, if any pooling equilibrium survives the Intuitive Criterion, the Riley equilibrium is defeated in the sense of Mailath, Okuno-Fujiwara, and Postlewaite (1993). ${ }^{35}$

\section{Conclusion}

We have analyzed a labor market where a worker's ability can be revealed either by his education or by his performance on-the-job. The addition of this realistic feature causes the failure of standard arguments, such as the selection of the Riley equilibrium via the Intuitive Criterion. Available evidence on the speed of employer learning suggests that this observation could be highly relevant for applied work in job market signaling and signaling models in general.

Our findings are also relevant for empirical research on signaling theory. Given the existence and stability of multiple pooling outcomes when learning is efficient, tests based on the properties of separating equilibria are likely to be too restrictive. ${ }^{36}$

We see two avenues for future research. The first is purely game-theoretic. As argued above, forward-looking equilibrium refinements beyond the Intuitive Criterion might not be entirely suitable to signaling models with receiver learning after the signaling stage, which raises a number of theoretical questions on refinements. The second concerns the learning process itself, whose characteristics can differ from one specific model (value function) to another. The general model presented in this paper abstracts from prevalent features of labor markets. Among other simplifications, it ignores the importance of match-specific uncertainty. This additional source of noise hampers signal extraction and is thus likely to expand the parameter space where the Intuitive Criterion bites. Another implicit premise of our analysis is that wages are a function of current beliefs. This is no longer true when employers can commit to employment contracts. Changing the perspective, it would be interesting to see whether commitment reinforces the informativeness of education signals.

Such extensions would provide a more realistic description of how signaling operates in labor markets. Our basic findings, however, apply to any signaling environment, beyond the particular jobmarket model we have focused on. This suggests plenty of scope for further research on the interactions between learning and signaling.

\footnotetext{
${ }^{35}$ Observe, however, that any pooling equilibrium with $e>0$ is defeated by the pooling equilibrium with $e=0$. Hence, in our framework, in general there will be defeated equilibria which survive the Intuitive Criterion.

${ }^{36}$ Empirical tests based on structural models have relied on Informational equilibria (Riley, 1979a; Riley, 1979b; Kaymak, 2006) rather than Perfect Bayesian equilibria. Proposition 5 may help providing such tests with a game theoretic foundation because Informational equilibria can be observationally similar to the equilibria with multiple pooling described in Proposition 5.
} 


\section{APPENDIX}

\section{Proof of Proposition 1:}

The wage is a linear function of current belief given by $w(\mu)=\mu\left(p_{h}-p_{l}\right)+p_{l}$. Hence, the value function reads

$$
v(p, \mu \mid r)=\frac{p_{l}}{r}+\frac{p_{h}-p_{l}}{1+r}\left(\mu+\frac{E\left[\mu^{\prime} \mid p, \mu\right]}{r}\right) .
$$

The boundary conditions in $\mathrm{P} 1$ follow from $E\left[\mu^{\prime} \mid p, \mu\right]=\mu$ when $\mu \in\{0,1\}$. To establish the ranking of the value functions it is sufficient to show that $E\left[\mu^{\prime} \mid p_{l}, \mu\right]<\mu<E\left[\mu^{\prime} \mid p_{h}, \mu\right]$ for all $\mu \in(0,1)$. We start by considering the first inequality. Let $\Lambda \equiv \mu /(1-\mu)$ and $X$ denote the output realization in period 1. Bayes rule implies that $\Lambda^{\prime}(X)=\Lambda\left[g_{h}(X) / g_{l}(X)\right]$ so that

$$
E\left[\Lambda^{\prime} \mid p_{l}, \Lambda\right]=\Lambda \int\left(\frac{g_{h}(X)}{g_{l}(X)}\right) g_{l}(X) d X=\Lambda .
$$

Given that $\Lambda$ is a convex function of $\mu$, Jensen's inequality yields $E\left[\mu^{\prime} \mid p_{l}, \mu\right]<\mu$. A similar reasoning using the converse transformation $\widetilde{\Lambda} \equiv(1-\mu) / \mu$ yields $E\left[\mu^{\prime} \mid p_{h}, \mu\right]>\mu$.

\section{Proof of Proposition 2:}

The wage does not directly depend on the worker's type, but solely on the current belief ratio $\theta$. It is equal to the expected output $E[p \mid \theta]=\left(p_{h}-p_{l}\right)\left(\frac{\theta}{1+\theta}\right)+p_{l}$.

For a low ability worker, $d \theta_{t}=\theta_{t} s d Z_{t}$ and thus the asset value solves the following HamiltonJacobi-Bellman equation

$$
r v\left(p_{l}, \theta\right)-\frac{1}{2}(\theta s)^{2} v^{\prime \prime}\left(p_{l}, \theta\right)=\left(p_{h}-p_{l}\right)\left(\frac{\theta}{1+\theta}\right)+p_{l},
$$

which is a second order non-homogeneous ODE with non-constant coefficients. The homogeneous problem satisfies an Euler equation ${ }^{37}$ whose solution reads

$$
v^{H}\left(p_{l}, \theta\right)=C_{1 l} \theta^{\alpha^{-}}+C_{2 l} \theta^{\alpha^{+}}
$$

where $\alpha^{-}$and $\alpha^{+}$are the negative and positive roots of the quadratic equation

$$
\alpha(\alpha-1) \frac{s^{2}}{2}-r=0 .
$$

Thus $\alpha^{-}=\frac{1}{2}(1-\Delta)$ and $\alpha^{+}=\frac{1}{2}(1+\Delta)$ with $\Delta=\frac{1}{s} \sqrt{s^{2}+8 r}$. Notice that $\alpha^{+}-\alpha^{-}=\Delta$ and $\alpha^{+}+\alpha^{-}=1$.

\footnotetext{
${ }^{37}$ Euler equations are second order homogeneous ODE of the form $\beta v(\theta)+\alpha \theta v^{\prime}(\theta)+\theta^{2} v^{\prime \prime}(\theta)=0$, for given constants $\beta$ and $\alpha$. They admit a closed form solution as described in e.g. Polyanin and Zaitsev (2003).
} 
To solve for the non-homogeneous equation we use the method of variations of parameters. The non-homogeneous term is composed of a non-linear function of $\theta$ plus a constant term. Thus we can assume that the particular solution is of the form

$$
v^{N H}\left(p_{l}, \theta\right)=\left[y_{1}(\theta) \theta^{\alpha^{-}}+y_{2}(\theta) \theta^{\alpha^{+}}\right]+\frac{p_{l}}{r} .
$$

Standard derivations yield the system of equations

$$
\left(\begin{array}{cc}
\theta^{\alpha^{-}} & \theta^{\alpha^{+}} \\
\alpha^{-} \theta^{\alpha^{-}-1} & \alpha^{+} \theta^{\alpha^{+-1}}
\end{array}\right)\left(\begin{array}{c}
y_{1}^{\prime}(\theta) \\
y_{2}^{\prime}(\theta)
\end{array}\right)=\left(\begin{array}{c}
0 \\
-\frac{2 \sigma}{(1+\theta) \theta s}
\end{array}\right) .
$$

Given that the Wronskian of the two linearly independent solutions is

$$
\theta^{\alpha^{-}} \alpha^{+} \theta^{\alpha^{+}-1}-\theta^{\alpha^{+}} \alpha^{-} \theta^{\alpha^{-}-1}=\alpha^{+}-\alpha^{-}=\Delta
$$

we have

$$
y_{1}(\theta)=\frac{2 \sigma}{s \Delta} \int \frac{1}{(1+x) x^{\alpha^{-}}} d x \quad \text { and } \quad y_{2}(\theta)=\frac{2 \sigma}{s \Delta} \int \frac{1}{(1+x) x^{\alpha^{+}}} d x .
$$

Thus the general form of the particular solution reads

$$
v^{N H}\left(p_{l}, \theta\right)=\frac{2 \sigma}{s \Delta}\left(\theta^{\alpha^{-}} \int \frac{1}{(1+x) x^{\alpha^{-}}} d x+\theta^{\alpha^{+}} \int \frac{1}{(1+x) x^{\alpha^{+}}} d x\right)+\frac{p_{l}}{r} .
$$

The bounds of integration and constants $C_{1 l}$ and $C_{2 l}$ of the homogeneous solution are pinned down by the boundary conditions

$$
v\left(p_{l}, \theta\right) \underset{\theta \rightarrow 0}{\longrightarrow} \frac{p_{l}}{r} \quad \text { and } \quad v\left(p_{l}, \theta\right) \underset{\theta \rightarrow \infty}{\longrightarrow} \frac{p_{h}}{r} .
$$

Let us first consider the homogeneous solution. Given that $\theta^{\alpha^{-}} \rightarrow \infty$ as $\theta \downarrow 0$, the first boundary condition can be satisfied if and only if $C_{1 l}$ equals zero. Similarly, because $\theta^{\alpha^{+}} \rightarrow \infty$ as $\theta \uparrow \infty$, the second boundary condition allows us to set $C_{2 l}$ equal to zero. All that remains is to determine the integration bounds in equation (6). Consider the following function

$$
v\left(p_{l}, \theta\right)=\frac{2 \sigma}{s \Delta}\left(\theta^{\alpha^{-}} \int_{0}^{\theta} \frac{1}{(1+x) x^{\alpha^{-}}} d x+\theta^{\alpha^{+}} \int_{\theta}^{\infty} \frac{1}{(1+x) x^{\alpha^{+}}} d x\right)+\frac{p_{l}}{r} .
$$

Let us examine first the limit when $\theta \downarrow 0$. Given that $\theta^{\alpha^{-}} \rightarrow \infty$ and $\int_{0}^{\theta}\left[(1+x) x^{\alpha^{-}}\right]^{-1} d x \rightarrow$ 0 as $\theta \downarrow 0$, we can apply l'Hôpital's rule to determine the limit. Straightforward calculations show that $\theta^{\alpha^{-}} \int_{0}^{\theta}\left[(1+x) x^{\alpha^{-}}\right]^{-1} d x \rightarrow-\theta /\left[(1+\theta) \alpha^{-}\right] \rightarrow 0$ as $\theta \downarrow 0$. A similar argument yields 
$\theta^{\alpha^{+}} \int_{\theta}^{\infty}\left[(1+x) x^{\alpha^{+}}\right]^{-1} d x \rightarrow \theta /\left[(1+\theta) \alpha^{+}\right] \rightarrow 0$ as $\theta \downarrow 0 .{ }^{38}$ Hence, (8) satisfies the first boundary condition in (7). Now, consider the limit when $\theta \uparrow \infty$. We can again use l'Hôpital's rule because $\theta^{\alpha^{-}} \rightarrow 0$ and $\int_{0}^{\theta}\left[(1+x) x^{\alpha^{-}}\right]^{-1} d x \rightarrow \infty$ as $\theta \uparrow \infty$, so that $\theta^{\alpha^{-}} \int_{0}^{\theta}\left[(1+x) x^{\alpha^{-}}\right]^{-1} d x \rightarrow-1 / \alpha^{-}$as $\theta \uparrow \infty$. Similarly, we obtain $\theta^{\alpha^{+}} \int_{\theta}^{\infty}\left[(1+x) x^{\alpha^{+}}\right]^{-1} d x \rightarrow 1 / \alpha^{+}$as $\theta \uparrow \infty$. Thus we have

$$
\lim _{\theta \rightarrow \infty} v\left(p_{l}, \theta\right)=\frac{2 \sigma}{s \Delta}\left(\frac{1}{-\alpha^{-}}+\frac{1}{\alpha^{+}}\right)+\frac{p_{l}}{r}=\frac{2 \sigma}{s}\left(\frac{-1}{\alpha^{-} \alpha^{+}}\right)+\frac{p_{l}}{r}=\frac{p_{h}}{r},
$$

where the last equality follows from $\alpha^{-} \alpha^{+}=-2 r / s^{2}$. Hence, we have established that (8) also satisfies the second boundary condition in (7), which completes the derivation of $v\left(p_{l}, \theta\right)$.

The asset value of the high type is derived similarly. For a high ability worker, $d \theta_{t}=\theta_{t} s\left(s d t+d Z_{t}\right)$ and thus the asset value solves

$$
r v\left(p_{h}, \theta\right)-\theta s^{2} v^{\prime}\left(p_{h}, \theta\right)-\frac{1}{2}(\theta s)^{2} v^{\prime \prime}\left(p_{h}, \theta\right)=\left(p_{h}-p_{l}\right)\left(\frac{\theta}{1+\theta}\right)+p_{l} .
$$

The homogeneous solution reads

$$
v^{H}\left(p_{h}, \theta\right)=C_{1 h} \theta^{\gamma^{-}}+C_{2 h} \theta^{\gamma^{+}},
$$

where $\gamma^{-}$and $\gamma^{+}$are the negative and positive roots of the quadratic equation

$$
\gamma(\gamma+1) \frac{s^{2}}{2}-r=0
$$

so that $\gamma^{-}=\frac{1}{2}(-1-\Delta)$ and $\gamma^{+}=\frac{1}{2}(-1+\Delta)$. The non-homogeneous solution is of the form

$$
v^{N H}\left(p_{h}, \theta\right)=\left[z_{1}(\theta) \theta^{\gamma^{-}}+z_{2}(\theta) \theta^{\gamma^{+}}\right]+\frac{p_{l}}{r},
$$

where the functions $z_{1}(\theta)$ and $z_{2}(\theta)$ satisfy

$$
\left(\begin{array}{cc}
\theta^{\gamma^{-}} & \theta^{\gamma^{+}} \\
\gamma^{-} \theta^{\gamma^{-1}} & \gamma^{+} \theta^{\gamma^{+-1}}
\end{array}\right)\left(\begin{array}{c}
z_{1}^{\prime}(\theta) \\
z_{2}^{\prime}(\theta)
\end{array}\right)=\left(\begin{array}{c}
0 \\
-\frac{2 \sigma}{(1+\theta) \theta s}
\end{array}\right) .
$$

Following the same steps as before yields the solution in Proposition 2.

We now show that the value function obtained above exhibits strong learning. We establish each property in turn.

\footnotetext{
${ }^{38}$ Notice that $\int_{\theta}^{\infty}\left[(1+x) x^{\alpha^{+}}\right]^{-1} d x<\int_{\theta}^{\infty} x^{-\alpha^{+}}-1 d x=\theta^{-\alpha^{+}} / \alpha^{+}$. Thus $\int_{\theta}^{\infty}\left[(1+x) x^{\alpha^{+}}\right]^{-1} d x$ is bounded for all $\theta>0$ and the asset equation is well defined.
} 
P1. This property is most easily established reversing the change of variable from $\theta_{t}$ to $\mu_{t}$

(i) Low ability worker : $d \mu_{t}=\mu_{t}\left(1-\mu_{t}\right) s\left(-s \mu_{t} d t+d Z_{t}\right)$,

(ii) High ability worker : $d \mu_{t}=\mu_{t}\left(1-\mu_{t}\right) s\left(s\left(1-\mu_{t}\right) d t+d Z_{t}\right)$.

By definition

$$
\begin{aligned}
v\left(p_{i}, \mu_{t}\right) & =E\left[\int_{t}^{+\infty} e^{-r(\tau-t)} w\left(\mu_{\tau}\right) d \tau \mid p_{i}, \mu_{t}\right] \\
& =\int_{t}^{+\infty} e^{-r(\tau-t)} E\left[w\left(\mu_{\tau}\right) \mid p_{i}, \mu_{t}\right] d \tau, \text { for all } \mu_{t} \in(0,1) \text { and } i \in\{l, h\}
\end{aligned}
$$

where the second equality follows from Fubini's theorem. When the worker is of the high type $\left(p_{i}=p_{h}\right)$, we know from (10) that $\mu_{t}$ has a positive deterministic trend: $\mu_{t}\left(1-\mu_{t}\right)^{2} s^{2}$. As $w\left(\mu_{t}\right)=\mu_{t}\left(p_{h}-p_{l}\right)+p_{l}$ is a linear function of $\mu_{t}$, it follows that $E\left[w\left(\mu_{\tau}\right) \mid p_{h}, \mu_{t}\right]>w\left(\mu_{t}\right)$ for all $\tau>t$, and so $v\left(p_{h}, \mu_{t}\right)>$ $w\left(\mu_{t}\right) / r$. Similarly, condition (9) shows that $\mu_{t}$ has a negative deterministic trend when the worker is of the low type, hence $v\left(p_{l}, \mu_{t}\right)<w\left(\mu_{t}\right) / r$. Finally, notice that when $\mu_{t}$ goes to zero or one, its stochastic component vanishes which provides us with the two boundary conditions.

P2. Differentiating (11) with respect to $s$ yields

$$
\frac{\partial v\left(p_{i}, \mu_{t}\right)}{\partial s}=\left(p_{h}-p_{l}\right) \int_{t}^{+\infty} e^{-r(\tau-t)} \frac{\partial E\left[\mu_{\tau} \mid p_{i}, \mu_{t}\right]}{\partial s} d \tau, \text { for all } \mu_{t} \in(0,1) \text { and } i \in\{l, h\} .
$$

It is therefore sufficient to prove that $\partial E\left[\mu_{\tau} \mid p_{i}, \mu_{t}\right] / \partial s$ is positive when $p_{i}=p_{h}$ and negative when $p_{i}=p_{l}$. This follows from (9) and (10) as beliefs exhibit a negative trend for low types and a positive trend for high types, both of them being increasing in absolute values with respect to $s$.

P3. The limit condition as $s \rightarrow 0$ is satisfied because both deterministic and stochastic terms in (9) and (10) converge to 0. Accordingly, beliefs remain constant, i.e. $\lim _{s \rightarrow 0} E\left[\mu_{\tau} \mid p_{i}, \mu_{t}\right]=\mu_{t}$ for $i \in\{l, h\}$. To establish the limit condition as $s \rightarrow \infty$, let us focus first on high types. Notice that the belief ratio $\theta_{t}$ is a geometric Brownian motion and so $\theta_{\tau}\left(Z \mid p_{h}, \theta_{t}\right)=\theta_{t} \exp \left(\frac{s^{2}}{2}(\tau-t)+s Z\right)$, where $Z$ is normally distributed with mean 0 and variance $\sigma^{2}(\tau-t)$. Given that $\mu(\theta)=\theta /(1+\theta)$, we have: $\mu_{\tau}\left(Z \mid p_{h}, \mu_{t}\right)=$ $\left[1-\mu_{t}+\mu_{t} \exp \left(-\frac{s^{2}}{2}(\tau-t)-s Z\right)\right]^{-1}$. Hence, for all $\varepsilon>0$ and $Z$, there exists a signal/noise ratio $\underline{s}(\varepsilon, Z)$ such that $1-\mu_{\tau}\left(Z \mid p_{h}, m u_{t}\right)<\varepsilon$ for all $s>\underline{s}(\varepsilon, Z)$. It follows that $\lim _{s \rightarrow \infty} E\left[\mu_{\tau} \mid p_{h}, \mu_{t}\right]=1$ which in turn implies that $\lim _{s \rightarrow \infty} v\left(\mu \mid p_{h}\right)=p_{h} / r$. One can establish in a similar fashion that $\lim _{s \rightarrow \infty} E\left[\mu_{\tau} \mid p_{l}, \mu_{t}\right]=0$ because $\mu_{\tau}\left(Z \mid p_{l}, \mu_{t}\right)=\left[1-\mu_{t}+\mu_{t} \exp \left(\frac{s^{2}}{2}(\tau-t)-s Z\right)\right]^{-1}$. 


\section{Proof of Proposition 3:}

In any separating equilibrium, $e_{l}=0$. For, if $e_{l}>0$, signaling will never lead to a wage below $p_{l} / r$ (by P1) but $c\left(e_{l}, p_{l}\right)>c\left(0, p_{l}\right)$ because costs are strictly increasing in the level of education.

In a separating equilibrium, each type must prefer its own education level to mimicking that of the other type. This leads to the inequalities

$$
c\left(p_{l}, e_{h}\right)-c\left(p_{l}, 0\right) \geq \frac{1}{r}\left(p_{h}-p_{l}\right) \geq c\left(p_{h}, e_{h}\right)-c\left(p_{h}, 0\right) .
$$

Given that cost functions are strictly increasing in $e$, there exists a unique $\underline{e}_{h}>0$ such that the first inequality is fulfilled if and only if $e_{h} \geq \underline{e_{h}}$, and a unique $\overline{e_{h}}>0$ such that the second inequality is fulfilled if and only if $e_{h} \leq \overline{e_{h}}$. By continuity, the condition given in the proposition uniquely determines those two education levels. Further, by the single crossing property

$$
c\left(p_{l}, \overline{e_{h}}\right)-c\left(p_{l}, 0\right)>c\left(p_{h}, \overline{e_{h}}\right)-c\left(p_{h}, 0\right),
$$

so that $\overline{e_{h}}>\underline{e_{h}}$. This shows that the set of equilibrium candidates is not empty and that all separating equilibria fulfill the specified conditions.

It remains to show that any pair $\left(e_{l}, e_{h}\right)$ fulfilling those conditions can be sustained in a separating equilibrium. For that, it suffices to specify the out-of-equilibrium beliefs. It is straightforward to check that the beliefs given by e.g $\mu(e)=1$ if $e \geq e_{h}$ and $\mu(e)=0$ if $e<e_{h}$ sustain such a profile.

\section{Proof of Proposition 4:}

We first prove that the given education levels can be sustained as a pooling equilibrium by the particular beliefs $\mu\left(e_{p}\right)=\mu_{0}$ and $\mu(e)=0$ for all $e \neq e_{p}$. These beliefs together with $e_{p}=0$ lead to pooling because any deviation yields a strictly larger cost and a strictly lower value (recall that the value function is strictly increasing in $\mu$ ). Consider $e_{p}>0$ : Under the stated beliefs, selecting $e=0$ is always preferred by both types to selecting any other $e \notin\left\{0, e_{p}\right\}$, for both yield the same lifetime income but the cost of $e=0$ is strictly smaller. Hence it suffices to check that neither of the two types has an incentive to deviate from $e_{p}$ to $e=0$. This yields the conditions

$$
c\left(p_{l}, e_{p}\right)-c\left(p_{l}, 0\right) \leq v\left(p_{l}, \mu_{0}\right)-\frac{p_{l}}{r} \quad \text { and } \quad c\left(p_{h}, e_{p}\right)-c\left(p_{h}, 0\right) \leq v\left(p_{h}, \mu_{0}\right)-\frac{p_{l}}{r} .
$$

By the single crossing property, $c\left(p_{h}, e_{p}\right)-c\left(p_{h}, 0\right)<c\left(p_{l}, e_{p}\right)-c\left(p_{l}, 0\right)$. Weak learning, however, implies that $v\left(p_{h}, \mu_{0}\right) \geq v\left(p_{l}, \mu_{0}\right)$ and so the first condition implies the second one. Given that costs are strictly increasing in education, there exists a unique $\overline{e_{p}}>0$ such that the first inequality is fulfilled if and only if $e_{p} \leq \overline{e_{p}}$. By continuity, that education level is uniquely determined by the condition given in the statement. It is straightforward to show that an education level can be sustained as 
a pooling equilibrium under some belief system if and only if it can be sustained under the beliefs specified above. Further, by P1

$$
v\left(p_{l}, \mu_{0}\right)-p_{l} \leq w\left(\mu_{0}\right)-p_{l}=\mu_{0}\left(p_{h}-p_{l}\right)<p_{h}-p_{l},
$$

which, recalling the definition of $\underline{e_{h}}$ in Proposition 3, implies that $\overline{e_{p}}<\underline{e_{h}}$.

Last, assume strong learning. To see that the set of pooling equilibria shrinks as the speed of learning increases, simply notice that $v\left(p_{l}, \mu_{0} \mid r, s\right)$ is strictly decreasing in $s$ by $\mathrm{P} 2$, hence the conclusion follows from the equation defining $\overline{e_{p}}$. The fact that $\lim _{s \rightarrow \infty} \overline{e_{p}}=0$ under strong learning follows from P3, i.e. the requirement that $\lim _{s \rightarrow \infty} v\left(p_{l}, \mu \mid r, s\right)=p_{l} / r$.

\section{Proof of Proposition 5:}

Suppose that there are at least two education levels in the support of the low types' equilibrium strategy, $e$ and $e^{\prime}$ with $e<e^{\prime}$. As both must be optimal, it follows that

$$
v\left(p_{l}, \mu(e)\right)-c\left(p_{l}, e\right)=v\left(p_{l}, \mu\left(e^{\prime}\right)\right)-c\left(p_{l}, e^{\prime}\right)
$$

which, since $c\left(p_{l}, e\right)<c\left(p_{l}, e^{\prime}\right)$, implies $v\left(p_{l}, \mu(e)\right)<v\left(p_{l}, \mu\left(e^{\prime}\right)\right)$. Hence, $\mu(e)<\mu\left(e^{\prime}\right)$ (recall that $v$ is strictly increasing in $\mu$ ), proving that $\mu$ needs to be strictly increasing over chosen education levels. This is only possible if $\mu\left(e^{\prime}\right)>0$, hence in equilibrium $e^{\prime}$ must also be in the support of the high types' strategy. It follows that there exist at most one education level chosen only by low types, and it must be the lowest one in the support of their strategy. A symmetric argument holds for high types, thereby establishing (i) and (ii). The fact that $e_{l}=0$ follows as in Proposition 3.

Suppose that also $e$ is in the support of the high types' strategy. Then

$$
v\left(p_{h}, \mu(e)\right)-c\left(p_{h}, e\right)=v\left(p_{h}, \mu\left(e^{\prime}\right)\right)-c\left(p_{h}, e^{\prime}\right) .
$$

By the single crossing property, we obtain

$$
v\left(p_{h}, \mu\left(e^{\prime}\right)\right)-v\left(p_{h}, \mu(e)\right)=c\left(p_{h}, e^{\prime}\right)-c\left(p_{h}, e\right)<c\left(p_{l}, e^{\prime}\right)-c\left(p_{l}, e\right)=v\left(p_{l}, \mu\left(e^{\prime}\right)\right)-v\left(p_{l}, \mu(e)\right) .
$$

The strict inequality leads to a contradiction in Spence's model because $v\left(p_{h}, \mu\right)=v\left(p_{l}, \mu\right)$. One can then conclude that low types randomize among at most two education levels, where the lower one is only chosen by them. Symmetrically, high types randomize among at most two education levels, where the higher one is only chosen by them.

For other value functions, the last equality does not lead to a contradiction. The example in the main text shows that equilibria where $E_{p}$ is not a singleton are indeed possible. 


\section{Proof of Proposition 8:}

By continuity, there exists $\varepsilon>0$ small enough that

$$
c\left(p_{l}, e\right)-c\left(p_{l}, e_{p}\right)<\frac{1}{2} \frac{p_{h}-p_{l}}{r} \quad \forall e \in\left(e_{p}, e_{p}+\varepsilon\right) .
$$

Let $\delta=c\left(p_{h}, e_{p}+\varepsilon\right)-c\left(p_{h}, e_{p}\right)>0$, so that $c\left(p_{h}, e\right)-c\left(p_{h}, e_{p}\right)>\delta$ for all $e>e_{p}+\varepsilon$. For a value function with strong learning,

$$
\lim _{s \rightarrow \infty} v\left(p_{l}, \mu \mid r, s\right)=\frac{p_{l}}{r} \quad \text { and } \quad \lim _{s \rightarrow \infty} v\left(p_{h}, \mu \mid r, s\right)=\frac{p_{h}}{r} .
$$

Hence there exists $s^{*}$ such that, for all $s \geq s^{*}$,

$$
\frac{p_{h}}{r}-v\left(p_{l}, \mu \mid r, s\right)>\frac{1}{2} \frac{p_{h}-p_{l}}{r} \quad \text { and } \quad \frac{p_{h}}{r}-v\left(p_{h}, \mu \mid r, s\right)<\frac{1}{2} \delta .
$$

It follows that, for $s \geq s^{*}$,

$$
c\left(p_{l}, e\right)-c\left(p_{l}, e_{p}\right)<\frac{p_{h}}{r}-v\left(p_{l}, \mu \mid r, s\right) \quad \forall e \in\left(e_{p}, e_{p}+\varepsilon\right),
$$

i.e. (ED) fails, implying that $e^{*}\left(e_{p}\right)>e_{p}+\varepsilon$, and

$$
c\left(p_{h}, e\right)-c\left(p_{h}, e_{p}\right)>\frac{p_{h}}{r}-v\left(p_{h}, \mu \mid r, s\right) \quad \forall e>e_{p}+\varepsilon,
$$

i.e. (PD) fails, implying that $e^{* *}\left(e_{p}\right)<e_{p}+\varepsilon$. We conclude that $e^{* *}\left(e_{p}\right)<e^{*}\left(e_{p}\right)$, or that the considered pooling equilibrium survives the Intuitive Criterion.

\section{Proof of Proposition 9:}

In this proof, we make the dependence of all involved quantities on $s$ explicit. Recall from Proposition 4 that pooling equilibria correspond to education levels in $\left[0, \overline{e_{p}}(s)\right]$ and that, under strong learning, $\overline{e_{p}}(s)$ is strictly decreasing in $s$ and $\lim _{s \rightarrow \infty} \overline{e_{p}}(s)=0$.

Recall also conditions (ED) and (PD). A pooling equilibrium with education level $e_{p}$ fulfills the Intuitive Criterion if and only if condition (PD) fails at $e=e^{*}\left(e_{p}\right)$, where the latter education level is defined by taking equality in condition (ED).

Define

$$
I_{h}\left(e_{p} \mid s\right)=c\left(p_{h}, e_{p}\right)-c\left(p_{h}, e^{*}\left(e_{p}\right)\right)+\frac{p_{h}}{r}-v\left(p_{h}, \mu_{0} \mid r, s\right) .
$$

It follows that the Intuitive Criterion fails at $e_{p}$ if and only if $I_{h}\left(e_{p} \mid s\right)>0$.

Step 1. $I_{h}$ is strictly increasing in $e_{p}$. Hence, for a given speed of learning $s$, if the Intuitive criterion fails at $e_{p}$, it also fails at all larger education levels. 
To prove this, differentiate the equality defining $e^{*}\left(e_{p}\right)$ to obtain

$$
\frac{\partial e^{*}\left(e_{p} \mid s\right)}{\partial e_{p}}=\frac{c_{e}\left(p_{l}, e_{p}\right)}{c_{e}\left(p_{l}, e^{*}\left(e_{p}\right)\right)}>0 .
$$

Now, differentiating $I_{h}$ with respect to $e_{p}$ yields

$$
\begin{aligned}
\frac{\partial I_{h}\left(e_{p} \mid s\right)}{\partial e_{p}} & =c_{e}\left(p_{h}, e_{p}\right)-c_{e}\left(p_{h}, e^{*}\left(e_{p}\right)\right) \cdot \frac{\partial e^{*}\left(e_{p} \mid s\right)}{\partial e_{p}} \\
& =\frac{c_{e}\left(p_{h}, e_{p}\right) c_{e}\left(p_{l}, e^{*}\left(e_{p}\right)\right)-c_{e}\left(p_{h}, e^{*}\left(e_{p}\right)\right) c_{e}\left(p_{l}, e_{p}\right)}{c_{e}\left(p_{l}, e^{*}\left(e_{p}\right)\right)}>0,
\end{aligned}
$$

where the last inequality follows from log-submodularity.

Step 2. There exists $s^{*}(0)$ such that all pooling equilibria fail the Intuitive Criterion for $s<s^{*}(0)$, and $e_{p}=0$ survives it for $s \geq s^{*}(0)$.

As in the proof of Proposition 8, we can find a speed of learning $s^{*}(0)$ such that $e_{p}=0$ survives the Intuitive Criterion for $s \geq s^{*}(0)$ and fails it for $s<s^{*}(0)$. In the latter case, the conclusion follows from Step 1.

Step 3. Let $s \geq s^{*}(0)$. There exists a unique education level $\widetilde{e}(s)$ such that $I_{h}(\widetilde{e}(s))=0$ and $I_{h}\left(e_{p}\right)>0$ for all $e_{p}>\widetilde{e}(s)$. Further, $\widetilde{e}(s)$ is strictly increasing in $s$ and $\lim _{s \rightarrow \infty} \widetilde{e}(s)=+\infty$.

It follows from Step 1 that either $I_{h}\left(e_{p}\right)>0$ for all $e_{p}$, and hence all pooling equilibria fail the Intuitive Criterion, or there exists a unique education level $\widetilde{e}(s)$ as stated. By Step 2, the former case can only occur if $s<s^{*}(0)$.

Notice that

$$
\frac{\partial I_{h}\left(e_{p} \mid s\right)}{\partial s}=-c_{e}\left(p_{h}, e^{*}\left(e_{p} \mid s\right)\right) \cdot \frac{\partial e^{*}\left(e_{p} \mid s\right)}{\partial s}-\frac{\partial v\left(p_{h}, \mu_{0} \mid r, s\right)}{\partial s}<0,
$$

where the inequality follows from P2 and the strictly decreasing profile of $e^{*}\left(e_{p} \mid s\right)$ in $s$ (see equality in condition (ED)).

Differentiating $I_{h}(\widetilde{e}(s))=0$ with respect to $s$ now yields

$$
\frac{\partial I_{h}\left(e_{p} \mid s\right)}{\partial e_{p}} \frac{\partial \widetilde{e}(s)}{\partial s}+\frac{\partial I_{h}\left(e_{p} \mid s\right)}{\partial s}=0
$$

which, since $\frac{\partial I_{h}\left(e_{p} \mid s\right)}{\partial e_{p}}>0$ and $\frac{\partial I_{h}\left(e_{p} \mid s\right)}{\partial s}<0$, implies $\frac{\partial \widetilde{e}(s)}{\partial s}>0$.

It follows that $\widetilde{e}(s)$ is a strictly increasing function. Hence, either $\lim _{s \rightarrow \infty} \widetilde{e}(s)=+\infty$ as claimed, or it has an upper bound and hence (by virtue of being increasing) a finite limit $L$. Suppose the latter case would hold. Recall that $e^{*}\left(e_{p}\right)>e_{p}$ for all $e_{p}$. As $I_{h}(\widetilde{e}(s) \mid s)=0$, from the definition of $I_{h}$ we obtain that

$$
\lim _{s \rightarrow \infty} c\left(p_{h}, e^{*}(\widetilde{s})\right)-c\left(p_{h}, \widetilde{e}(s)\right)=0
$$


because $\lim _{s \rightarrow \infty} v\left(p_{h}, \mu \mid r, s\right)=\frac{p_{h}}{r}$ in the strong learning case. This is a contradiction with $e^{*}(L)>L$. Step 4. For $s \geq s^{*}(0)$, the rest of the proof follows.

Recapitulating, $e_{p}$ yields a pooling equilibrium if and only if $e_{p} \in\left[0, \overline{e_{p}}(s)\right]$, and in that case survives the Intuitive Criterion if and only if $e_{p} \in[0, \widetilde{e}(s)]$. Given that $\overline{e_{p}}(s)$ is strictly decreasing and converges to 0 as $s \rightarrow \infty$, and $\widetilde{e}(s)$ is strictly increasing and diverges to infinity (and $\widetilde{e}\left(s^{*}(0)\right)=0<\overline{e_{p}}\left(s^{*}(0)\right)$ ), it follows that they must intersect at a unique speed of learning $\bar{s}$ such that part (b) holds below $\bar{s}$ and part (c) holds above it.

It remains to consider the case where $c_{e}$ is log-linear. Retracing our steps, we can see from the computations in Step 1 that, in this case, $\frac{\partial I_{h}}{\partial e_{p}}=0$, while from Step 2 we still see that $\frac{\partial I_{h}}{\partial s}<0$. It follows that, for a fixed $s$, either all pooling equilibria fail the Intuitive criterion, or all survive it (the locus of $\widetilde{s}$ becomes vertical).

\section{References}

Altonji, J., And C. Pierret (2001): "Employer Learning and Statistical Discrimination," Quarterly Journal of Economics, 116(1), 313-350.

Banks, J. S., And J. Sobel (1987): "Equilibrium Selection in Signaling Games," Econometrica, $55(3), 647-662$.

Cho, I.-K., And D. M. Kreps (1987): "Signaling Games and Stable Equilibria," Quarterly Journal of Economics, 102(2), 179-221.

Chung, K. L., And R. J. Williams (1990): Introduction to Stochastic Integration, 2nd Ed. Birkhaüser, Boston.

Daley, B., And B. Green (2007): "Market Signaling with Grades," Mimeo, Stanford University.

Esö, P., And J. Schummer (2008): "Credible Deviations from Signaling Equilibria," Mimeo, Northwestern University.

Farber, H. S., and R. Gibbons (1996): "Learning and Wage Dynamics," Quarterly Journal of Economics, 111(4), 1007-47.

Feltovich, N., R. Harbaugh, and T. To (2002): "Too Cool for School? Signalling and Countersignalling," RAND Journal of Economics, 33(4), 630-649.

Fudenberg, D., And J. Tirole (1991): Game Theory. The MiT Press, Cambridge, Massachusetts. 
Gibbons, R., And L. F. Katz (1991): "Layoffs and Lemons," Journal of Labor Economics, 9(4), $351-80$.

Habermalz, S. (2006): "The Speed of Employer Learning and Job Market Signaling Revisited," IZA Discussion Paper.

Jovanovic, B. (1979): "Job Matching and the Theory of Turnover," Journal of Political Economy, 87(5), 972-990.

Kaymak, B. (2006): "Signaling, Self-Selection, and Employer Learning," Mimeo, Stanford University.

Kohlberg, E., and J.-F. Mertens (1986): "On the Strategic Stability of Equilibria," Econometrica, 54(5), 1003-37.

Kreps, D., and R. Wilson (1982): "Sequential Equilibrium," Econometrica, 50(4), 863-894.

LANGe, F. (2007): "The Speed of Employer Learning," Journal of Labor Economics, 25(1), 1-35.

Mas-Colell, A., M. D. Whinston, and J. R. Green (1995): Microeconomic Theory. Oxford University Press, Oxford (UK).

Moscarini, G. (2005): "Job Matching and the Wage Distribution," Econometrica, 73(2), 481-516.

Polyanin, A. D., And V. F. Zaitsev (2003): Handbook of Exact Solutions for Ordinary Differential Equations, 2nd Ed. Chapman and Hall/CRC Press, Boca Raton.

Riley, J. G. (1979a): "Informational Equilibrium," Econometrica, 47(2), 331-360.

- (1979b): "Testing the Educational Screening Hypothesis," Journal of Political Economy, 87(5), S227-S252.

Spence, M. (1973): “Job Market Signaling," Quarterly Journal of Economics, 87(3), 355-374. 